\title{
Nobel Lecture: On superconductivity and superfluidity (what I have and have not managed to do) as well as on the "physical minimum" at the beginning of the XXI century*
}

\author{
Vitaly L. Ginzburg ${ }^{\dagger}$ \\ P.N. Lebedev Physics Institute, Russian Academy of Sciences, 119991 Moscow, Russian \\ Federation
}

(Published 2 December 2004)

\section{INTRODUCTION}

First of all I would like to express my heartfelt gratitude to the Swedish Royal Academy of Sciences and its Nobel Committee for physics for awarding me the 2003 Nobel Prize in physics. I am well aware of how difficult it is to select no more than three Laureates out of the far greater number of nominees. So all the more valuable is this award. Personally, I have two additional reasons for appreciating this award. First, I am already 87, the Nobel Prize is not awarded posthumously, and posthumous recognition is not all that significant to me since $\mathrm{I}$ am an atheist. Second, the 1958 and 1962 Nobel Prizes were awarded, respectively, to Igor' Evgen'evich Tamm and Lev Davidovich Landau. Outside of high school, the title of "teacher" quite often describes a formal relationship: for instance, it is applied to the supervisor in the preparation of a thesis. But I believe that your real teachers are those who have made the greatest impact on your work and whose example you have followed. Tamm and Landau (see Figs. 1 and 2) were precisely these kind of people for me. I feel particularly pleased, because in a sense I have justified their faith in me. Of course, the reason lies not with the Prize itself, but with the fact that my receiving the award after them signifies that I have followed their path.

Now about the Nobel Lecture. It is the custom, I do not know whether by rule or natural tradition, that the Nobel Lecture is concerned with the work for which the Prize was awarded. But I am aware of at least one exception. P. L. Kapitza was awarded the 1978 Prize for "his basic inventions and discoveries in the area of lowtemperature physics" But Kapitza's Lecture was entitled "Plasma and the Controlled Thermonuclear Reactions." He justified his choice of topic as follows: he had worked

\footnotetext{
*The 2003 Nobel Prize in Physics was shared by A. A. Abrikosov, Vitaly L. Ginzburg, and Anthony J. Leggett. This lecture is the text of Professor Ginzburg's address on the occasion of the award.

${ }^{\dagger}$ Electronic address: ginzburg@lpi.ru; translated by E. N. Ragozin, edited by K. Franchuk and K. Friedman.
}

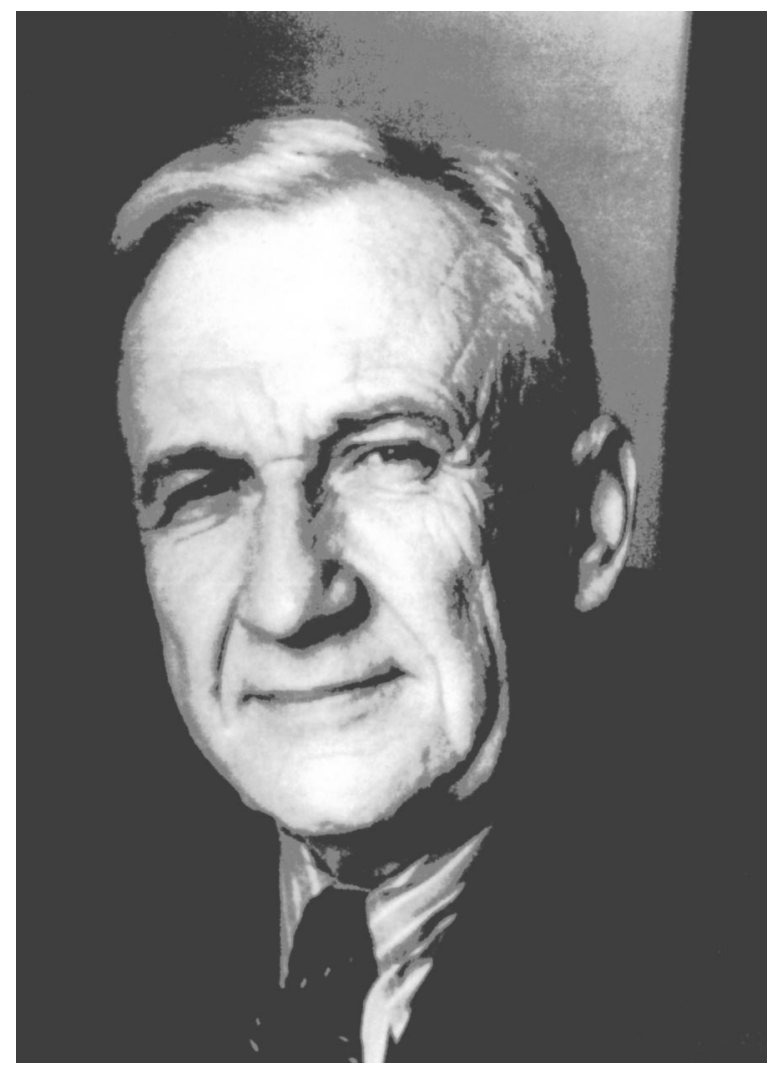

FIG. 1. Igor' Evgen'evich Tamm, 1895-1971.

in the field of low-temperature physics many years before he had been awarded the Prize and he believed it would be more interesting to speak of what he was currently engaged in. That is why P. L. Kapitza spoke of his efforts to develop a fusion reactor employing highfrequency electromagnetic fields. By the way, this path has not led to success, which is insignificant in the present context.

I have not forgotten my "pioneering contributions to the theory of superconductors and superfluids" for which I have received the Prize, but I would like not to dwell on them. The point is that in 1997 I decided to sum up my activities in that field, and I wrote a paper entitled 


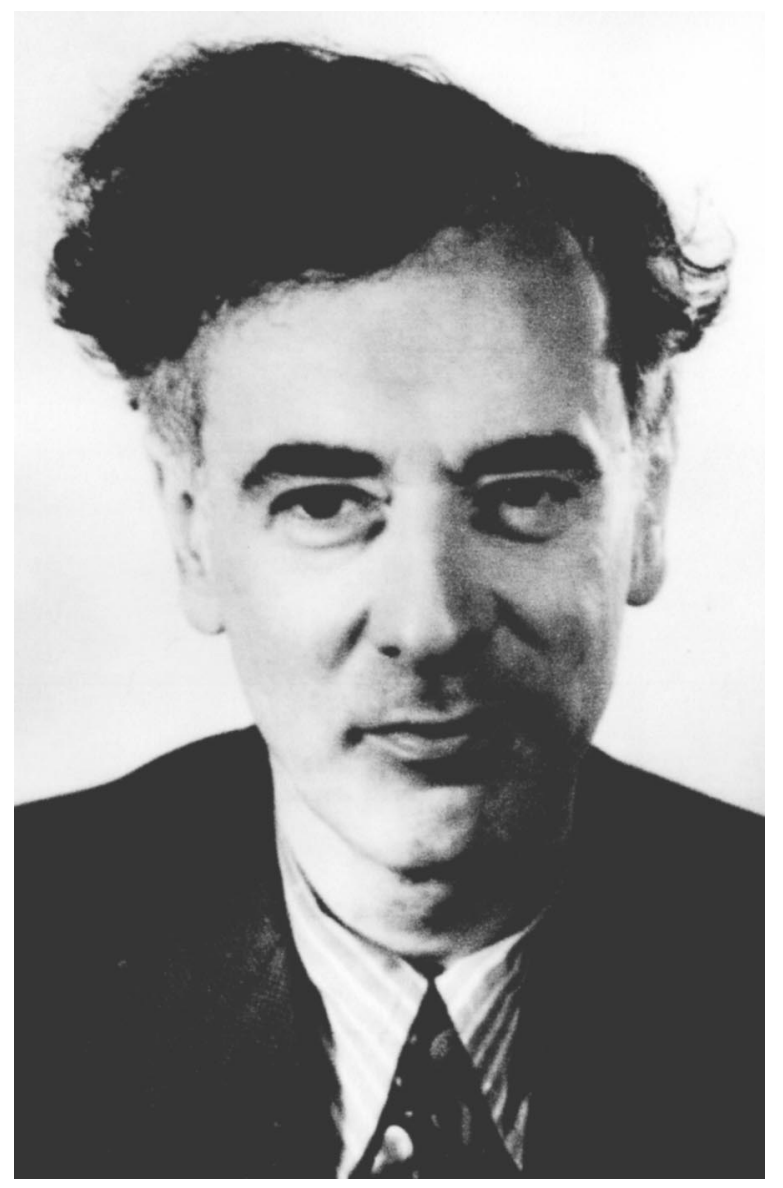

FIG. 2. Lev Davidovich Landau, 1908-1968.

"Superconductivity and superfluidity (what was and what was not done)" (Ginzburg, 1997, 1998). ${ }^{1}$ In particular, this article set out in detail the story of quasiphenomenological superconductivity theory, as constructed jointly with Landau (Ginzburg and Landau, 1950). Under the circumstances, it would be unnecessary, and above all tedious, to repeat all that. Furthermore, the Ginzburg-Landau theory of superconductivity, which I call the $\Psi$ theory of superconductivity, is employed in the work of A. A. Abrikosov (Abrikosov, 1957), and he will supposedly discuss it in his Nobel Lecture. This is to say nothing of the fact that the $\Psi$ theory of superconductivity has been covered in many books [see, for instance, Lifshitz and Pitaevskii (1978); Tinkham (1996)]. At the same time, there are several problems bearing on the field of superconductivity and superfluidity which I have taken up and which have not been adequately investigated. This is why I decided to focus on two of these important problems in my lecture.

The two issues I would like to address here are ther-

\footnotetext{
${ }^{1}$ Article 7 in Ginzburg (2003) is the somewhat edited article referenced here.
}

moelectric effects in the superconducting state and the $\Psi$ theory of superfluidity. However, before I turn to these issues, I will nevertheless give some background on my activities in the field of superconductivity. At the end of the lecture I will allow myself to touch on an educational program for physicists (the issue of a "physical minimum"), which has been of interest to me for more than 30 years.

\section{BRIEF ACCOUNT OF MY ACTIVITY IN THE FIELD OF SUPERCONDUCTIVITY PRIOR TO THE ADVENT OF HIGH-TEMPERATURE SUPERCONDUCTORS}

Lev Landau was in prison for exactly one year and was released on April 28, 1939 primarily due to the efforts of Kapitza, who became his "personal guarantee". 2 Landau remained in this situation (of needing a guarantor) until his premature death in 1968. The Landau "case" was officially discharged for lack of a "corpus delicti" (evidence that a crime had been committed) only in 1990(!). The imprisonment had a strong effect on Landau, but fortunately it did not bereave him of his outstanding capabilities as a physicist. That is why he "justified the confidence," as they said at that time, of those who released him on bail instead of shooting him or leaving him to rot in jail (Landau personally told me that he had not been far from death) by constructing his superfluidity theory (Landau, 1941). I was present at his report on this topic in 1940 or maybe in 1941 (the paper was submitted for publication on May 15, 1941). Also considered at the end of this paper was superconductivity, which was treated as the superfluidity of electron liquid in metals.

That work impressed me, of course, but at that time I was enthusiastic about quite a different set of questions, namely, the theory of higher-spin particles. That is why I did not take up the low-temperature subject right away, and shortly after our lives radically changed when the war broke out (as is well known, for the USSR it began on June 22, 1941). The Physical Institute of the USSR Academy of Sciences, where I was working and still work, was evacuated from Moscow to the town of Kazan, where many difficulties were encountered, which I describe in my autobiography. In any case, it was not until 1943 that I made an attempt to do, in the spirit of the Landau theory of superfluidity (Landau, 1941), something of the same kind applied to superconductivity. ${ }^{3}$ That work (Ginsburg, 1944a) is of no great value today, but I believe there were some interesting points in it, for Bardeen considered it at length in his famous review (Bardeen, 1956). Even then I was aware the work was poor and therefore did not submit it to a journal in English, which we would normally have

\footnotetext{
${ }^{2}$ For more details, see, for example, article 10 in Ginzburg (2003)

${ }^{3}$ True, somewhat earlier I had considered the problem of light scattering in helium II (Ginzburg, 1943) on the basis of the Landau theory (Landau, 1941).
} 


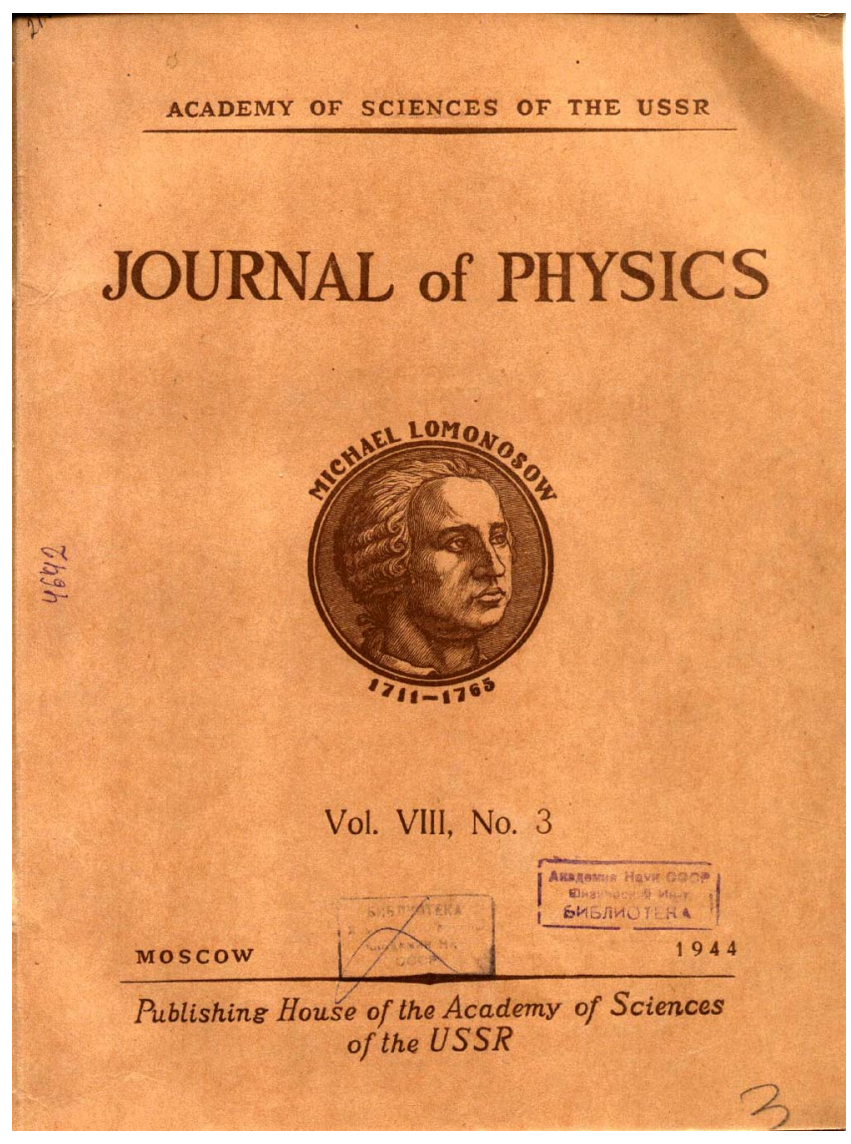

FIG. 3. (Color in online edition) The English-language journal by which much Russian Physics work reached the West.

done at that time [the journal-Journal of Physics USSR (see Fig. 3) - was terminated in 1947 during the cold war]. My next paper was concerned with thermoelectric effects in the superconducting state (Ginsburg, 1944b), and its destiny seems to be unusual and strange. Sixty years have passed, but some predictions made in that work have never been verified and thermoelectric effects in the superconducting state have not been adequately investigated. I myself returned to these problems more than once, but made no significant progress. Appeals addressed to other physicists have only a minor effect, for the problem is out of fashion. Here I would like to take advantage of my last opportunity to bring it to the attention of the physics community. Section IV below is concerned with this question.

However, the question of thermoelectric effects in superconductors, while interesting, is still a particular problem, which clearly emerges only in the presence of a temperature gradient. Furthermore, at that time there existed no thorough theory of superconductivity even under thermodynamic equilibrium. The fact is that the well-known London theory advanced in 1935 (London and London, 1935a, 1935b) (which will also be discussed in Sec. IV of this lecture) yielded much, and is widely employed under certain conditions even today (Lifshitz and Pitaevskii, 1978; Tinkham, 1996; Waldram, 1996), but it is insufficient. In my next work, performed as far back as 1944 (Ginzburg, 1946a), I explored why the Lon- don theory is inapplicable in a strong magnetic field (in the theory of superconductivity, the field is termed strong when it is on the order of the critical magnetic field $H_{\mathrm{c}}$; we are dealing with type-I superconductors). From the London theory it follows also that the surface energy at the interface between the normal and superconducting phases is negative, and to attain positiveness one is forced to introduce without substantiation some additional and, moreover, high surface energy of nonelectromagnetic origin. Therefore it became evident that the London theory had to be generalized. This problem was solved in 1950 in the $\Psi$ theory of superconductivity (Ginzburg and Landau, 1950). ${ }^{4}$ This brings up the question, which has been repeatedly addressed to me: why did it take five years after the work of Ginzburg (1946a), in which the necessity of generalizing the London theory was recognized, to construct the $\Psi$ theory? Of course, I cannot answer this question as regards other physicists. As to myself, to some extent I was nearing my objective, as described in Ginzburg $(1997,1998)$. But, I believe, the main reason for the slowness of this process lay with the fact that I did not focus my attention on the theory of superconductivity. Theoretical physicists have the good fortune to be able to work almost simultaneously in different directions and in general to move from one subject to another. Specifically, in the period from 1944 to 1950, apart from superconductivity and superfluidity, I was engaged in studying radio-wave propagation in the ionosphere (plasma), radio-frequency solar radiation, light scattering in liquids, the theory of transition radiation (I. M. Frank and I jointly called attention to the existence of this effect), the relativistic theory of higherspin particles (in part jointly with Tamm), undulator radiation, the theory of ferroelectrics, and other things. Of special note is the fact that my work on ferroelectric effects (as applied primarily to $\mathrm{BaTiO}_{3}$ ) considered them (Ginzburg, 1945) on the basis of the Landau theory of phase transitions, and this approach subsequently made progress [see article 5 in Ginzburg (2003)]. . $^{5}$

The $\Psi$ theory of superconductivity (Ginzburg and Landau, 1950) is, if you like, an application of the Landau theory of phase transitions to superconductivity. In this case, some scalar complex $\Psi$ function fulfils the role of the order parameter. Here I restrict myself to giving the equations employed for $\Psi$ and the vector electromagnetic field potential A (as is generally known, $\operatorname{rot} \mathbf{A}=\mathbf{H}$, where $\mathbf{H}$ is the magnetic-field strength, which does not differ from the magnetic induction $\mathbf{B}$ in this

\footnotetext{
${ }^{4}$ As already mentioned, this theory is commonly referred to as the Ginzburg-Landau theory. But I use the term $\Psi$ theory of superconductivity, because it seems to me that using one's own name sounds, at least in Russian, somewhat pretentious. Furthermore, a similar theory, as applied to suprefluidity, was jointly elaborated in my work not with Landau, but with L. P. Pitaevskii and A. A. Sobyanin.

${ }^{5}$ For more details on the above-mentioned and other works of mine, see the article "A Scientific Autobiography - An Attempt" in Ginzburg (2001).
} 
case; furthermore, advantage is taken of the gauge $\operatorname{div} \mathbf{A}=0)$ :

$$
\begin{aligned}
& \frac{1}{2 m^{*}}\left(-i \hbar \nabla-\frac{e^{*}}{c} \mathbf{A}\right)^{2} \Psi+\alpha \Psi+\beta|\Psi|^{2} \Psi=0, \\
& \Delta \mathbf{A}=-\frac{4 \pi}{c} \mathbf{j}_{\mathrm{s}}, \\
& \mathbf{j}_{\mathrm{s}}=-\frac{i e^{*} \hbar}{2 m^{*}}\left(\Psi^{*} \nabla \Psi-\Psi \nabla \Psi^{*}\right)-\frac{\left(e^{*}\right)^{2}}{m c}|\Psi|^{2} \mathbf{A} .
\end{aligned}
$$

We consider an equilibrium or, in any case, a stationary state, and we assume that the normal current density in the superconductor is $\mathbf{j}_{\mathrm{n}}=0$ (the total current density is $\mathbf{j}=\mathbf{j}_{\mathrm{s}}+\mathbf{j}_{\mathrm{n}}$, where $\mathbf{j}_{\mathrm{s}}$ is the superconducting current density). Furthermore, at the superconductor-vacuum interface we impose the boundary condition

$$
\mathbf{n}\left(-i \hbar \nabla-\frac{e^{*}}{c} \mathbf{A}\right) \Psi=0
$$

where $\mathbf{n}$ is the normal to the interface.

In the vicinity of the critical temperature $T_{c}$, at which there occurs the normal-to-superconducting phase transition in the equilibrium case, in the $\Psi$ theory it can (and even must) be assumed that

$$
\alpha=\alpha_{c}^{\prime}\left(T-T_{c}\right), \quad \beta=\beta\left(T_{c}\right) \equiv \beta_{c}>0, \quad \alpha_{c}^{\prime}>0
$$

and the superconductor behavior is determined by the parameters

$$
\delta_{0}=\sqrt{\frac{m^{*} c^{2} \beta_{c}}{4 \pi\left(e^{*}\right)^{2}|\alpha|}}, \quad \varkappa=\frac{m^{*} c}{e^{*} \hbar} \sqrt{\frac{\beta_{c}}{2 \pi}}=\frac{\sqrt{2} e^{*}}{\hbar c} H_{\mathrm{cm}} \delta_{0}^{2} .
$$

Here, $\delta_{0}$ is the depth of penetration of the weak magnetic field $H \ll H_{\mathrm{cm}}$, and $H_{\mathrm{cm}}$ is the critical magnetic field for massive samples (earlier, mention was made of the critical field $H_{\mathrm{c}}$, which, say, for films is stronger than $\left.H_{\mathrm{cm}}\right)$.

Since the $\Psi$ theory is phenomenological, the values of mass $m^{*}$ and charge $e^{*}$ are not known beforehand. In this case, since $\Psi$ is not an observable quantity (among the observable quantities are, in particular, the $\delta_{0}$ and $H_{\mathrm{cm}}$ quantities), the mass can be arbitrarily selected: it is not among the measurable (observable) quantities. The choice of the $e^{*}$ value is a very interesting and intriguing question. It seemed to me from the outset that $e^{*}$ is some effective charge, which may be different from the electron charge or, as is said on occasion, the free-electron charge $e$. However, Landau did not see why $e^{*}$ should be different from $e$, and in our paper (Ginzburg and Landau, 1950) we compromised by writing that "there are no grounds to believe that the charge $e^{*}$ is different from the electron charge." I remained of my opinion and saw that the way to solve this question was to compare the theory with experiment. Specifically, the charge $e^{*}$ enters in Eq. (5) for $\varkappa$, where $\delta_{0}$ and $H_{\mathrm{cm}}$ are measured by experiment; at the same time, $x$ enters into the expres- sion for the surface energy $\sigma_{\mathrm{ns}}$, for the depth of penetration in a strong field (the field $H \geqslant H_{\mathrm{cm}}$ ), and for the limiting fields of the overcooling and overheating of superconducting samples. Following the path of comparing the theory with experiment, I arrived at the conclusion (Ginsburg, 1955a) that $e^{*}=(2-3) e$. When I discussed this result with Landau, he raised an objection, which he had evidently been guided by before, though had not advanced it. Specifically, with the charge $e^{*}$ assumed to be an effective quantity like, say, the effective mass $m_{\text {eff }}$ in the theory of metals and semiconductors, the effective charge may, and generally speaking, will, depend on the coordinates, because the parameters that characterize the semiconductor are functions of the temperature, the pressure, and the composition, which in turn may depend on the coordinates $\mathbf{r}$. If $e^{*}(\mathbf{r})$, the gauge (gradient) invariance of Eq. (2) of the $\Psi$ theory is lost. I did not find objections to this remark, and in an article (Ginsburg, 1955a) outlined the situation (reporting Landau's opinion, naturally with his permission).

The solution, however, was quite simple. After the advent of the Bardeen-Cooper-Schrieffer (BCS) theory in 1957 (Bardeen et al., 1957), it became clear that in superconductors there occurs a pairing of electrons with opposite momenta and spins (I imply the simplest case). The resultant pairs, which are sometimes referred to as Cooper pairs, possess zero spin and are Bose particles or, to be more precise, quasiparticles. The Bose-Einstein condensation of these pairs is responsible for the origin of superconductivity. By the way, as early as 1952 I noted (Ginsburg, 1952a; Ginzburg, 1953) that the charged Bose gas would behave like a superconductor, but did not arrive at the idea of pairing. Interestingly, this idea had been advanced (Ogg, 1946a, 1946b; Schafroth, 1954, 1955) even before Cooper (Cooper, 1956). It is immediately apparent from the BCS theory that the role of charge in the theory of superconductivity should supposedly be played by the pair charge, i.e., $2 e$. This fact was proved by Gor'kov (1959a, 1959b), who derived the $\Psi$-theory equations from the BCS theory. Therefore Landau was right in the sense that the charge $e^{*}$ should be universal and I was right in that it is not equal to $e$. However, the seemingly simple idea that both requirements are compatible and $e^{*}=2 e$ occurred to none of us. After the event one may be ashamed of this blindness, but this is by no means a rare occasion in science, and it is not so much that $\mathrm{I}$ am ashamed as that $\mathrm{I}$ am rather disappointed I did not see the solution.

Many results were obtained in our work (Ginzburg and Landau, 1950). For small values of the parameter $\varkappa$ we calculated the surface energy $\sigma_{\text {ns }}$ and pointed out that it lowers with increasing $x$ and vanishes when $x$ $=x_{c}=1 / \sqrt{2}$. Relying on the available experimental data we believed that for pure superconductors $x<x_{c}$, and this is generally correct. In any case, we considered in detail only superconductors with $x<x_{c}$, which now are termed type-I superconductors. Subsequently I would also restrict myself to the investigation of type-I superconductors (a certain exception is Ginzburg, 1956a). In 

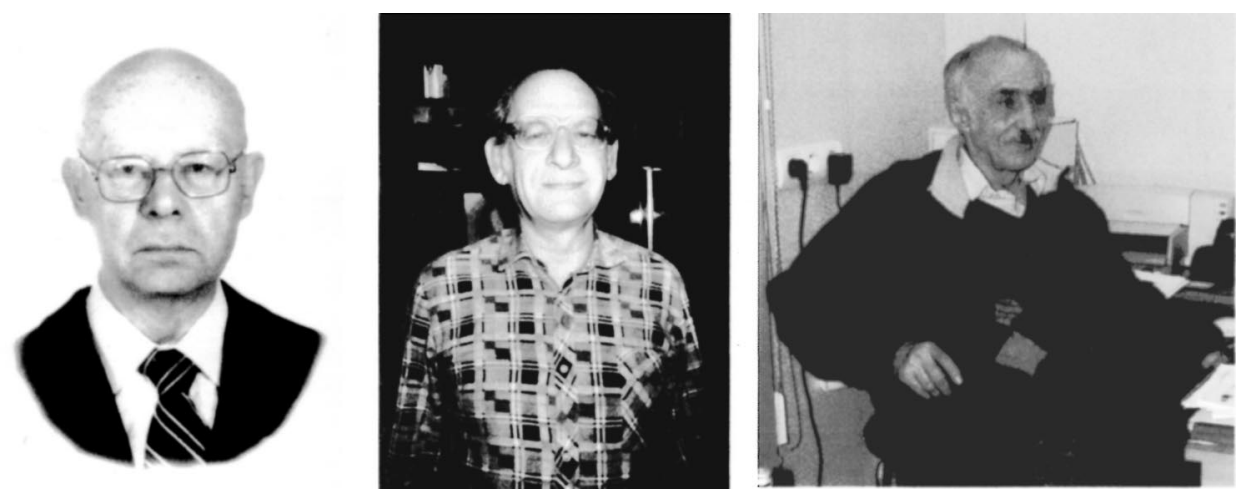

FIG. 4. Colleagues involved in work on superconductivity: G. F. Zharkov (1926-2004), D. A. Kirzhnits (1926-1998), and E. G. Maksimov.

1950, as well as previously, the superconducting alloys were known to usually behave in a significantly different manner from that of pure superconductors. Particularly clear data concerning alloys were obtained by L. V. Shubnikov ${ }^{6}$ and his collaborators in Kharkov in the mid 1930s (see references and the results in Shoenberg, 1965; this material was also touched upon by Ginzburg, 1946b; for more details see Buckel, 1972). In Buckel (1972), use is made of the term "Shubnikov phase" for the alloys investigated by Shubnikov. However, an understanding of the situation was lacking, and Landau and I, like many others, believed that alloys were an "unsavory business" and did not take an interest in them, restricting ourselves to the materials with $x<x_{c}$ for which $\sigma_{\text {ns }}>0$, i.e., type-I superconductors. True, as noted in A. Abrikosov's paper (Abrikosov, 1957) and in Lifshitz and Pitaevskii (1978), Landau hypothesized that alloys have $x>1 / \sqrt{2}$, i.e., they are type-II superconductors according to present-day concepts.

The solution of different problems on the basis of $\Psi$-theory equations was our concern in the bulk of our work (Ginzburg and Landau, 1950). Apart from the above-mentioned question of the energy $\sigma_{\mathrm{ns}}$, we considered primarily the behavior of superconducting plates and films in the external magnetic field and in some cases in the presence of current, and in doing this compared the theory with experiment. Subsequently, Landau lost interest in such calculations and in general in the development of the $\Psi$ theory. My own efforts in this direction are described in Ginzburg $(1997,1998)$. Here, I restrict myself to the mention of a fairly evident yet important generalization to the anisotropic case of the $\Psi$ theory (Ginsburg, 1952b), in which superconductors were assumed to be isotropic (Ginzburg and Landau, 1950). I also explored the overheating and overcooling of superconductors in a magnetic field (Ginzburg, 1958) and the quantization of magnetic flux in the case of a superconducting cylinder with an arbitrary wall thickness (Ginzburg, 1962). After the construction of the BCS theory I compared the $\Psi$ theory with experiment

\footnotetext{
${ }^{6}$ In 1937, when Stalin's terror was in full swing, L. V. Shubnikov was arrested and shot.
}

(Ginzburg, 1959). Of special note is Ginzburg (1956b), which was further developed by Zharkov (1958, 1959; see Fig. 4). This article, which had little bearing on the $\Psi$ theory, applied to ferromagnetic superconductors, which had not yet been observed at that time, and it suggested that the explanation for this fact related to the inclusion of magnetic energy. Subsequently (after the construction of the BCS theory), it became clear that the emergence of superconductivity in ferromagnetics is also hampered due to spin interaction. I was not engaged in that problem, but did give consideration in Ginzburg (1956b) to the idea of changing the role of the magnetic factor by the use of thin films and materials with a relatively strong coercive force. I do not think that anyone has paid attention to these possibilities, for old papers are seldom read. Of course, I do not feel sure that at the present stage one can find something of interest in Ginzburg (1956b) or Zharkov (1958, 1959)-I would just like these papers to be looked at.

In long ago 1943, I engaged in the study of superconductivity because at that time this phenomenon appeared to be the most mysterious one in the physics of the condensed state. But after the construction of the $\Psi$ theory, and especially of the BCS theory, the picture generally became clear as regards the materials known at that time. That is why my interest in superconductivity decreased, though I worked in this area episodically (see, for instance, Ginzburg, 1960, 1962). My interest was rekindled in 1964 in connection with the problem of the feasibility of high-temperature superconductors. Mercury - the first superconductor discovered in 1911possesses $T_{c}=4.15 \mathrm{~K}$, while the boiling temperature of ${ }^{4} \mathrm{He}$ at atmospheric pressure is $T_{\mathrm{b},}{ }^{4} \mathrm{He}=4.2 \mathrm{~K}$. By the way, from 1908 to 1923, for 15 long years, liquid helium was obtained only in Leiden, and low-temperature physics research was pursued on a very small scale, judged by present-day standards. By way of example the bibliography given at the end of the monograph (Ginzburg, 1946b) contains about 450 references to papers on superconductivity (and a few related problems) over the period from 1911 to 1944; of these, only 35 references fall within the 1911-1925 period. In contrast, after 19861987, when high-temperature superconductivity was dis- 
covered, during the ten subsequent years approximately 50,000 papers were published, i.e., about 15 papers per day(!).

Immediately after the discovery and first investigations of superconductivity the question arose of why this phenomenon is observed only at low temperatures or, in other words, helium temperatures. Naturally, there was no way to provide the answer until the nature of superconductivity was understood, i.e., till the construction of the BCS theory in 1957 (Bardeen et al., 1957). The following expression was derived for the critical temperature in this theory:

$$
T_{\mathrm{c}}=\theta \exp \left(-\frac{1}{\lambda_{\text {eff }}}\right),
$$

where $k_{\mathrm{B}} \theta$ is the energy range near the Fermi energy $E_{\mathrm{F}}=k_{\mathrm{B}} \theta_{\mathrm{F}}$, in which the conduction electrons (more precisely, the corresponding quasiparticles) are attracted together, which is responsible for pair production and the instability of the normal state; furthermore, in the simplest case, $\lambda_{\text {eff }}=\lambda=N(0) V$, where $N(0)$ is the electronic level density near the Fermi surface in the normal state and $V$ is some average matrix element of electron interaction which corresponds to the attraction. In the BCS theory, in its initial form, the coupling constant $\lambda_{\text {eff }}$ and, specifically, $\lambda$ is assumed to be small ("weak coupling"), i.e.,

$$
\lambda \ll 1 \text {. }
$$

As regards the temperature $\theta$, in the BCS theory it was assumed that

$$
\theta \sim \theta_{\mathrm{D}},
$$

where $\theta_{\mathrm{D}}$ is the Debye temperature of the metal, for the interelectron attraction was thought to be due to electron-phonon interaction (as is generally known, the highest phonon energy in a solid is of the order of $k_{\mathrm{B}} \theta_{\mathrm{D}}$ ). Typically, $\theta_{\mathrm{D}} \lesssim 500 \mathrm{~K}$ and $\lambda \lesssim \frac{1}{3}$; whence it follows, according to Eq. (6), that $T_{\mathrm{c}} \lesssim 500 \exp (-3)=25 \mathrm{~K}$ or, more generally,

$$
T_{\mathrm{c}} \lesssim 30-40 \mathrm{~K} \text {. }
$$

Defining all this more precisely would be out of place here. But this will suffice to show why condition (9) is fulfilled for typical metals, and even safely fulfilled. In particular, prior to the discovery of high-temperature superconductivity in 1986-1987, all attempts to discover or produce a superconductor with the highest possible critical temperature had led to the production (in 1973) of only the $\mathrm{Nb}_{3} \mathrm{Ge}$ compound with $T_{\mathrm{c}}=23-24 \mathrm{~K}$ (of course, in what follows I do not endeavor to find the exact values of various parameters; they depend on the purity and processing of the samples, etc.).

\section{ON HIGH-TEMPERATURE AND ROOM-TEMPERATURE SUPERCONDUCTORS}

The advent of the BCS theory made it possible to envisage the feasibility of a radical elevation of the criti- cal temperature. It may be that I am not familiar with some facts, but to my knowledge this question was clearly and constructively posed for the first time by Little in 1964 (Little, 1964). Little suggested replacing the phonon mechanism of attraction between conduction electrons with the attraction arising from interaction with bound electrons present in the same system. I call this mechanism excitonic or electron-excitonic; that is, we are dealing with the replacement of phonons by excitons-excitations in the system of bound electrons. True, this term is not universally used in the literature. In his case, Little employed a quasi-one-dimensional model, in which some conducting "spine" was surrounded by side "polarizers," say, organic molecules. For electronic excitons or, in other words, for the excited states of bound electrons, the characteristic temperatures $\theta_{\mathrm{ex}}=E_{\mathrm{ex}} / k_{\mathrm{B}} \leq \theta_{\mathrm{F}} \sim 10^{4}-10^{5} \mathrm{~K}$ and, in any case, the values $\theta_{\mathrm{ex}} \sim 10^{4} \mathrm{~K}$ are quite realistic. It is therefore evident that replacing $\theta \sim \theta_{\mathrm{D}}$ in Eq. (6) with $\theta \sim \theta_{\mathrm{e} x}$ gives us the values $T_{\mathrm{c}} \lesssim 10^{3} \mathrm{~K}$ (when, say, $\lambda \sim \frac{1}{3}$ ). Of course, these are no more than words, for it is still unclear how to realize the Little model, and this has never been accomplished. Furthermore, it became clear that the fluctuations in quasi-one-dimensional systems are so strong that the transition to the superconducting state is unlikely to occur. However, having familiarized myself with the paper of Little (1964), I put forward straightaway (Ginzburg, 1964a, 1964b) a quasi-two-dimensional model, wherein a plane conductor is in contact with a dielectric, say, a dielectric film. We termed the development of this version-the alternation of thin conducting layers with dielectric layers-a "sandwich." Going over from the quasi-one-dimensional model to the quasi-twodimensional model was not accidental, for immediately before this work, D.A. Kirzhnits ${ }^{7}$ (see Fig. 4) and I had considered (Ginzburg and Kirzhnits, 1964), not in connection with the high-temperature superconductivity problem, the problem of two-dimensional (surface) superconductivity. By the way, this problem is still of interest in itself, but I cannot enlarge on it here, so I refer the reader to the original papers (Ginzburg, 1964a, 1964b, 1989a).

Compared to quasi-one-dimensional systems, quasitwo-dimensional systems have the advantage that they exhibit significantly weaker fluctuations that destroy superconductivity. We took up the quasi-two-dimensional version (Ginzburg, 1964a, 1964b; Ginzburg and Kirzhnits, 1967). More precisely, at FIAN (the P. N. Lebedev Physical Institute of the USSR Academy of Sciences) a group of theorists turned to the high-temperature superconductivity problem in the broad sense, considering all the issues and possibilities known to us. The fruits of this labor were represented in the monograph The Problem of High-Temperature Superconductivity (Ginzburg and Kirzhnits, 1977); even its English version (1982; see Fig. 5) appeared 4-5 years before the experimental realiza-

\footnotetext{
${ }^{7}$ Unfortunately, the outstanding theoretical physicist D. A. Kirzhnits met an untimely death in 1998.
} 


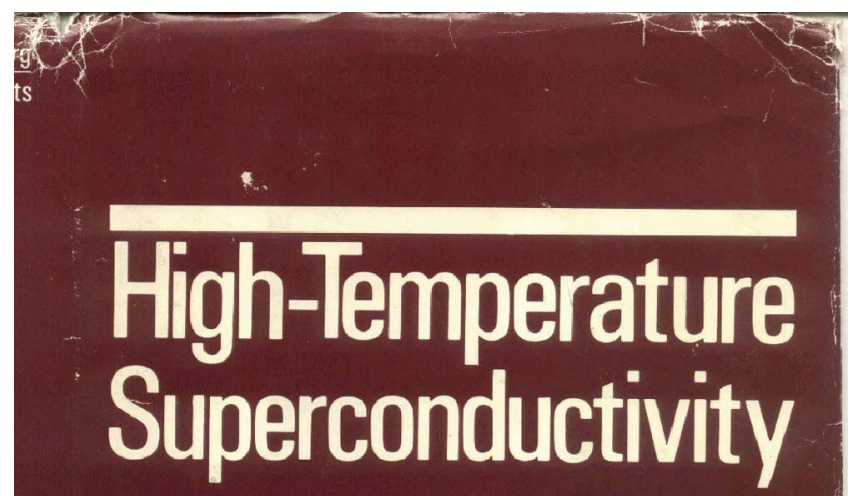

Edited by V. L. Ginzburg and D. A. Kirzhnits

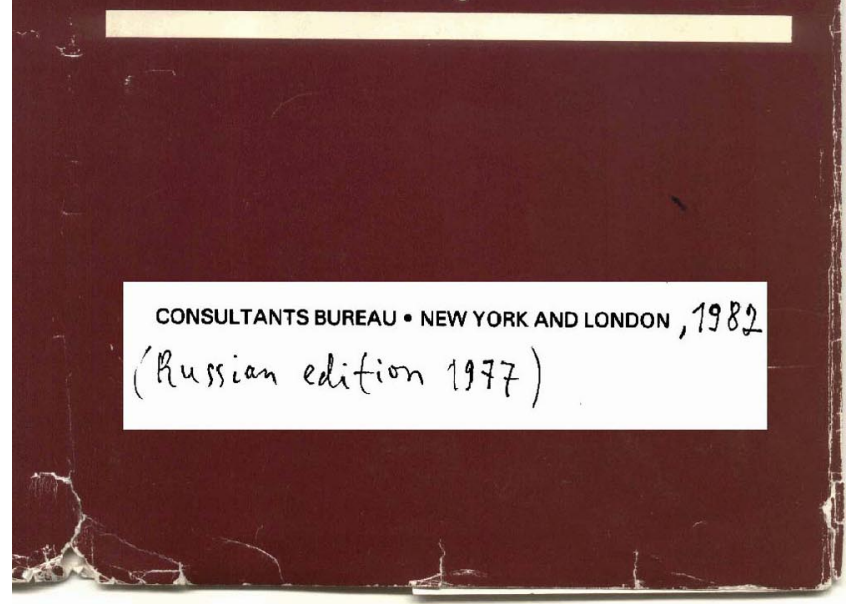

FIG. 5. (Color in online edition) The English translation in 1982 (Ginzburg and Kirzhnits, 1977) of a collection of articles by several contributors from the P. N. Lebedev Physical Institute of the USSR Academy of Sciences. The Russian edition was published in 1977.

tion of high-temperature superconductors (Bednorz and Muller, 1986; Wu et al., 1987) in 1986-1987. If the consideration of different models and possibilities is omitted, the most significant quantitative finding of our work, which is primarily due to Kirzhnits, is the crystal stability condition. The main argument against the possibility of developing a high-temperature superconductor was the anxiety that the crystal lattice would be unstable for the metal parameter values required to obtain it, i.e., for a material with $T_{\mathrm{c}}>T_{\mathrm{b}, N_{2}}=77.4 \mathrm{~K} .{ }^{8}$ When the problem is formulated in terms of the longitudinal material permittivity $\varepsilon(\omega, \mathbf{q})$, where $\omega$ is the frequency and $\mathbf{q}$ is the wave vector (we restrict our consideration to an isotropic body here), the production of electron pairs necessitates, roughly speaking, that the interelectron interaction $V=e^{2} / \varepsilon(0, q) r$ be negative, i.e., that it correspond to attraction. But this corresponds to the requirement that $\varepsilon(0, q)<0$. Meanwhile, based on other

\footnotetext{
${ }^{8} \mathrm{I}$ do not know whether there exists a commonly accepted definition of what can be regarded as a high-temperature superconductor. In my opinion, high-temperature superconductivity takes place when $T_{\mathrm{c}}>77.4 \mathrm{~K}$, i.e., is higher than the boiling temperature of nitrogen at atmopheric pressure.
}

considerations, it was believed that the lattice would be stable when

$$
\varepsilon(0, q)>0 .
$$

True, on closer examination (see Ginzburg, 1997, 1998; Ginzburg and Kirzhnits, 1977) it was found that superconductivity is also possible under condition (10), but the $T_{c}$ values would turn out to be moderate, even below the estimate (9). In Ginzburg and Kirzhnits (1977) and references therein it was found that the correct stability condition for $q \neq 0$ is of the form

$$
\frac{1}{\varepsilon(0, q)} \leqslant 1,
$$

i.e., stability is fulfilled when either of two inequalities,

$$
\varepsilon(0, q)>1, \quad \varepsilon(0, q)<0,
$$

takes place. In other words, any negative values of $\varepsilon(0, q)$ are admissible from the standpoint of stability, and there are no limitations on $T_{\mathrm{c}}$. To be more precise, up to now we do not know of such limitations. The following conclusion was drawn from our work, which is contained in Chapter 1 in the book The Problem of High-Temperature Superconductivity (Ginzburg and Kirzhnits, 1977):

On the basis of general theoretical considerations, we believe at present that the most reasonable estimate is $T_{\mathrm{c}} \lesssim 300 \mathrm{~K}$; this estimate being, of course, for materials and systems under more or less normal conditions (equilibrium or quasiequilibrium metallic systems in the absence of pressure or under relatively low pressures, etc.). In this case, if we exclude from consideration metallic hydrogen and, perhaps, organic metals, as well as semimetals in states near the region of electronic phase transitions, then it is suggested that we should use the exciton mechanism of attraction between the conduction electrons.

In this scheme, the most promising materialsfrom the point of view of the possibility of raising $T_{\mathrm{c}}$-are, apparently, layered compounds and dielectric-metal-dielectric sandwiches. However, the state of the theory, let alone the experiment, is still far from being such as to allow us to regard other possible directions as being closed, in particular, the use of filamentary compounds. Furthermore, for the present state of the problem of hightemperature superconductivity, the most sound and fruitful approach will be one that is not preconceived, in which attempts are made to move forward in the most diverse directions.

The investigation of the problem of hightemperature superconductivity is entering into the second decade of its history (if we are talking about the conscious search for materials with $T_{\mathrm{c}}$ $\geq 90 \mathrm{~K}$ with the use of the exciton and other mechanisms). Supposedly, there begins at the same time a new phase of these investigations, which is characterized not only by greater scope and diver- 
sity, but also by a significantly deeper understanding of the problems that arise. There is still no guarantee whatsoever that the efforts being made will lead to significant success, but a number of new superconducting materials have already been produced and are being investigated. Therefore it is, in any case, difficult to doubt that further investigations of the problem of high-temperature superconductivity will yield many interesting results for physics and technology, even if materials that remain superconducting at liquid nitrogen (or even room) temperatures will not be produced. Besides, as has been emphasized, this ultimate aim does not seem to us to have been discredited in any way. As may be inferred, the next decade will be crucial for the problem of high-temperature superconductivity.

This was written in 1976. Time passed, but the multiple attempts to find a reliable and reproducible way of creating a high-temperature superconductor were unsuccessful. As a result, after the flurry of activity came a falloff of interest which gave cause for me to characterize the situation in a popular paper (Ginzburg, 1984) published in 1984 as follows:

It has somehow happened that research into hightemperature superconductivity has become unfashionable (there is good reason to speak of fashion in this context since fashion sometimes plays a significant part in research work and in the scientific community). It is hard to achieve anything by making admonitions. Typically it is some obvious success (or reports of success, even if erroneous) that can radically and rapidly reverse attitudes. When they smell success, the former doubters, and even dedicated critics, are capable of an about-face and of becoming ardent supporters of the new work. But this subject belongs to the psychology and sociology of science and technology. In short, the search for high-temperature superconductivity can readily lead to unexpected results and discoveries, especially since the predictions of the existing theory are rather vague.

I did not expect, of course, that this "prediction" would come true in two years (Bedrnorz and Muller, 1986; Wu et al., 1987). It came true not only in the sense that high-temperature superconductors with $T_{\mathrm{c}}>T_{\mathrm{b}, N_{2}}$ $=77.4 \mathrm{~K}$ were obtained, but also, so to speak, in the social aspect: a real boom began and a "high-temperature superconductivity mania" started. One of the manifestations of the boom and the mania was the almost total oblivion of everything that had been done before 1986, as if the discussion of the high-temperature superconductivity problem had not begun 22 years before (Ginzburg 1964a, 1964b; Little, 1964). I have already dwelt on this subject above and in Ginzburg (1989b, 1996) and would not like to return to it here. An exception to this behavior was J. Bardeen, whom I have always respected, and who treated the high-temperature superconductivity problem with understanding both before and after 1986 (see Ginzburg, 1986; this article was also published in Ginzburg, 2001).

The foregoing in no way implies that our group or I pretend to a practical contribution of great importance to the development of high-temperature superconductivity. At the same time I believe that Little's works and ours did play a significant role in the formulation of the problem and drew attention to it. The solution of the problem was obtained to a large measure accidentally. The proposal to employ layered compounds was reasonable and promising, but neither I nor, to my knowledge, anybody else proposed the use of precisely the cuprates. Other layered compounds investigated do not belong to the high-temperature superconductors. The following fact serves to illustrate the accidental, to a certain extent, character of the discovery of high-temperature superconductivity. As far back as 1979, in one of the institutes in Moscow they produced and investigated (Shaplygin et al., 1979) a $\mathrm{La}_{1.8} \mathrm{Sr}_{0.2} \mathrm{CuO}_{4}$ ceramic, which was close to that investigated by Bednorz and Muller, with $T_{\mathrm{c}} \simeq 36 \mathrm{~K}$ (Cava et al., 1987). However, Shaplygin et al. (1979) measured the resistance of their samples at temperatures not lower than the liquid-nitrogen temperature and therefore did not discover their superconductivity. From the above one may draw a trivial conclusion that all newly produced materials should be tested for superconductivity. Also evident is another conclusion, namely, that even today it is possible to make a major discovery and next year be awarded a Nobel Prize for it without gigantic facilities and the work of a large group. This should be a source of inspiration, particularly for young people.

The present situation in condensed-matter theory does not allow us to calculate the value of $T_{\mathrm{c}}$ nor of other superconductor parameters, with the possible exception of a metallic hydrogen yet to be produced. Moreover, for more than 15 years the mechanism of superconductivity in the cuprates has remained obscure. I should remark that, despite the fact that I counted on the excitonic mechanism in high-temperature superconductivity research, the role of this mechanism in the known high-temperature superconductors is still completely unclear. In this case, in high-temperature superconductors (in cuprates) with $T_{\mathrm{c}}<170 \mathrm{~K}$ (the highestknown value $T_{\mathrm{c}} \simeq 165 \mathrm{~K}$ was attained back in 1994 in the $\mathrm{HgBa}_{2} \mathrm{Ca}_{2} \mathrm{Cu}_{3} \mathrm{O}_{8+x}$ cuprate under high pressure), as I see it, the electron-phonon mechanism of pairing may prove to be the dominant one. This possibility has previously been underestimated (in particular, by me), since the estimate (9) has served as a guide. But it is valid only for weak coupling (7). For a strong coupling (i.e., when $\lambda_{\text {eff }}$ $\gtrsim 1$ ), Eq. (6) is no longer applicable, but even from this formula it is clear that $T_{\mathrm{c}}$ increases with $\lambda_{\text {eff }}$.

The generalization of the BCS theory (Bardeen et al., 1957) to the strong-coupling case (Eliashberg, 1960a, $1960 \mathrm{~b})$ enables us to investigate the corresponding possibilities. Their analysis (see particularly Makimov, 2000, and references therein and Ginzburg, 1997, 1998) suggests that the electron-phonon mechanism in cuprates may well ensure superconductivity with $T_{\mathrm{c}} \lesssim 200 \mathrm{~K}$ ow- 
ing to the high $\theta_{\mathrm{D}}$ and $\lambda_{\text {eff }}$ values. At the same time, the electron-phonon interaction alone is supposedly insufficient in the context of so-called $d$ pairing and maybe other special features of superconductivity in cuprates. However, the role of other possibilities (spin interactions, excitonic interaction) is unclear. Of course, it would be out of place to discuss this vital topical problem here. I only want, on the one hand, to emphasize that the long-standing disregard of electron-phonon interactions in cuprates has always seemed, and still seems, unjustified to me (see Ginzburg and Maksimov, 1992). On the other hand, the likelihood of attaining, on the basis of the electron-phonon mechanism, the values $T_{\mathrm{c}} \sim 300 \mathrm{~K}$, and this is room-temperature superconductivity, appears to be small, as with the use of the spin mechanism. At the same time, the excitonic mechanism, as far as I know, does not provoke objections for $T_{\mathrm{c}}$ $\sim 300 \mathrm{~K}$, either. That is why I pin my hopes on precisely this mechanism for the attainment of room-temperature superconductivity. However, all this is no more than an intuitive judgment.

The development of high-temperature superconductivity was my dream for 22 years, even with no guarantee that the goal was at all attainable and, in particular, attainable in the foreseeable future. In my view, obtaining room-temperature superconductivity now occupies the same place.

\section{THERMOELECTRIC PHENOMENA IN THE SUPERCONDUCTING STATE}

The first attempt to observe thermoelectric phenomena and, specifically, thermoelectric current or thermal electromotive force in a nonuniformly heated circuit of two superconductors, to my knowledge, was made by Meissner (1927). He arrived at the conclusion that the thermoelectric effect is completely absent from superconductors. When I took an interest in this problem in 1943, this viewpoint was generally accepted [see, for instance, Burton et al. (1940) and especially the first and later editions of the book Superconductivity by Shoenberg (1965)]. However, I have encountered this assertion more recently as well. Nonetheless, this conclusion is erroneous, as I pointed out (Ginsburg, 1944b) as far back as 1944 (see Fig. 6).

The point is that the superconducting state can carry, apart from a superconducting current $\mathbf{j}_{\mathrm{s}}$, a normal current $\mathbf{j}_{\mathrm{n}}$ as well. This normal current is carried by "normal electrons," i.e., electron- or hole-type quasiparticles present in the metal in both the normal and superconducting states. In the superconducting state, the density of such normal quasiparticles depends strongly on the temperature and, generally, tends to zero as $T \rightarrow 0$. These notions, which are sometimes referred to as the two-liquid model, can be traced back to a paper by Gorter and Casimir (1934). An isotropic nonsuperconductor or, more precisely, an isotropic metal residing in a normal state, can carry only current with a density
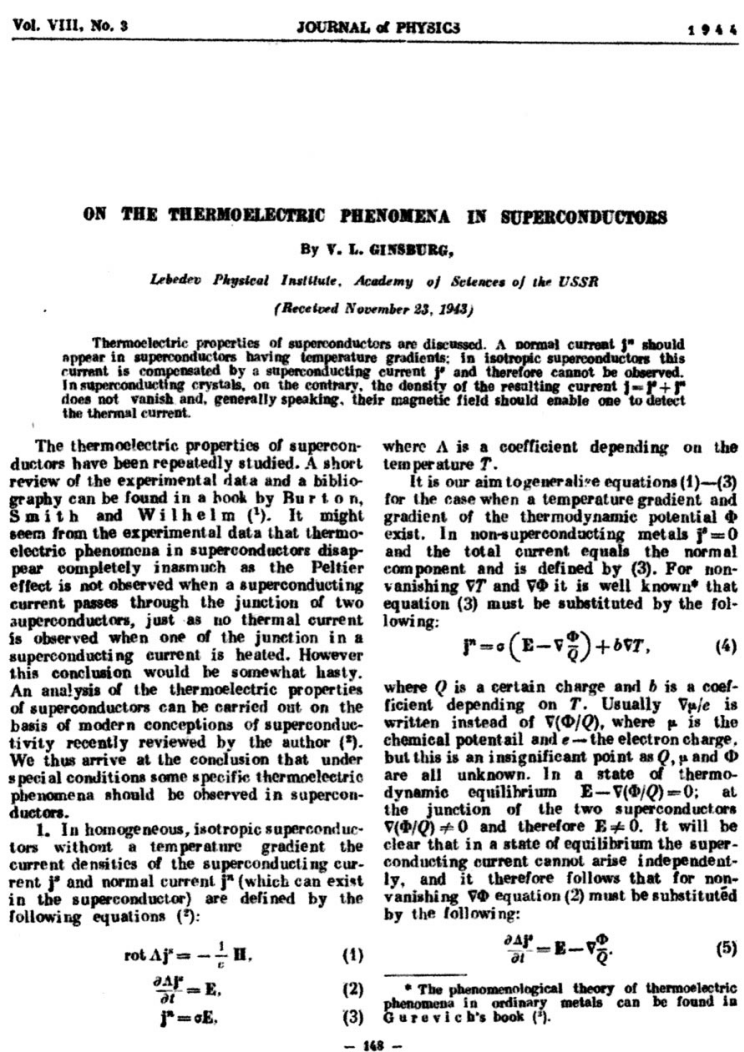

FIG. 6. Title page of Ginsburg (1944b).

$$
\mathbf{j}=\sigma\left(\mathbf{E}-\frac{\nabla \mu}{e}\right)+b \nabla T,
$$

where $\mu$ is the chemical potential of the electrons and $\mathbf{E}$ is the electric field. In the superconducting state, for a normal current we have (Ginzburg and Zharkov, 1978)

$$
\mathbf{j}_{\mathrm{n}}=\sigma_{\mathrm{n}}\left(\mathbf{E}-\frac{\nabla \mu}{e}\right)+b_{\mathrm{n}} \nabla T .
$$

At the same time, the superconducting current density $\mathbf{j}_{\mathrm{s}}$ in the London-theory approximation (London and London, 1935a, 1935b, 1944), to which we restrict ourselves here (naturally, this is precisely the approximation used in Ginsburg, 1944b), obeys the equations

$$
\begin{aligned}
& \operatorname{rot}\left(\Lambda \mathbf{j}_{\mathrm{s}}\right)=-\frac{1}{c} \mathbf{H}, \\
& \frac{\partial\left(\Lambda \mathbf{j}_{\mathrm{s}}\right)}{\partial t}=\mathbf{E}-\frac{\nabla \mu}{e},
\end{aligned}
$$

where $\Lambda=m /\left(e^{2} n_{\mathrm{s}}\right)$ is somewhat a constant, with $n_{s}$ being the "superconducting electron" density (so that $\mathbf{j}_{s}$ $=e n_{\mathrm{s}} \mathbf{v}_{\mathrm{s}}$, where $\mathbf{v}_{\mathrm{s}}$ is the velocity); in this scheme, the field penetration depth is

$$
\delta_{\mathrm{L}}=\sqrt{\frac{\Lambda c^{2}}{4 \pi}}=\sqrt{\frac{m c^{2}}{4 \pi e^{2} n_{s}}} .
$$

Notice that this is a simplification, as different chemical potentials $\mu_{\mathrm{n}}$ and $\mu_{\mathrm{s}}$ should in fact be introduced in Eqs. (14) and (16), respectively, for the normal and supercon- 


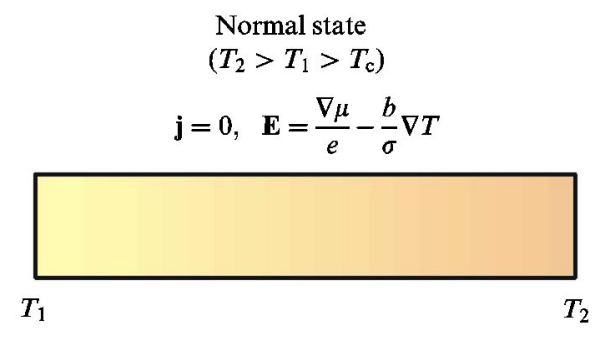

Superconducting state

b)

$$
\begin{gathered}
\left(T_{\mathrm{c}}>T_{2}>T_{1}\right) \\
\mathbf{j}=\mathbf{j}_{\mathrm{s}}+\mathbf{j}_{\mathrm{n}}=0 ; \mathbf{j}_{\mathrm{n}}=b_{\mathrm{n}} \nabla T, \mathbf{H}=0 \\
\mathbf{E}-\frac{\nabla \mu}{e}=0
\end{gathered}
$$

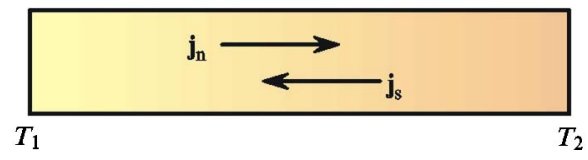

FIG. 7. (Color in online edition) Rod with ends at two different temperatures $T_{1}$ and $T_{2}$ : (a) normal state; (b) superconducting state. Thermoelectric current $\mathbf{j}_{\mathrm{n}}$ is present in the supercon-ducting state. According to Ginsburg, 1944a.

ducting electrons. In addition, yet another term (generally, not large) proportional to $\nabla j_{\mathrm{s}}^{2}$ figures in Eq. (16) (see Ginzburg and Zharkov, 1978). When the superconductor is nonuniform, the parameter $\Lambda$ depends on the coordinates.

As is clear from Eq. (16), in the stationary case, in the superconductor

$$
\mathbf{E}-\frac{\nabla \mu}{e}=0
$$

and, in view of Eq. (14),

$$
\mathbf{j}_{\mathrm{n}}=b_{\mathrm{n}}(T) \nabla T \text {. }
$$

Therefore, the thermoelectric current $\mathbf{j}_{\mathrm{n}}$ in no way vanishes in the superconducting state. However, this current is not directly observable in the simplest case, because it is compensated for by the superconducting current $\mathbf{j}_{\mathrm{s}}$. Let us consider a uniform superconducting rod, one end of which resides at a temperature $T_{2}$ and the other at a temperature $T_{1}<T_{2}$ (Fig. 7). Then, in the normal state (i.e., when $T_{1}>T_{\mathrm{c}}$ ), since there is no closed circuit, from Eq. (13) we have [see Fig. 7(a)]

$$
\mathbf{j}=0, \quad \mathbf{E}-\frac{\nabla \mu}{e}=-\frac{b}{\sigma} \nabla T .
$$

In the superconducting state (for $T_{2}<T_{c}$ ),

$$
\begin{aligned}
& \mathbf{j}=\mathbf{j}_{\mathrm{s}}+\mathbf{j}_{\mathrm{n}}=0, \quad \mathbf{j}_{\mathrm{s}}=-\mathbf{j}_{\mathrm{n}}=-b_{\mathrm{n}} \nabla T, \\
& \mathbf{H}=0, \quad \mathbf{E}-\frac{\nabla \mu}{e}=0 .
\end{aligned}
$$

True, near the rod ends, where $\mathbf{j}_{\mathrm{s}}$ transforms to $\mathbf{j}_{\mathrm{n}}$ or vice versa, uncompensated charges emerge (the charge imbalance effect) and therefore the field $\mathbf{E}$ is not equal to

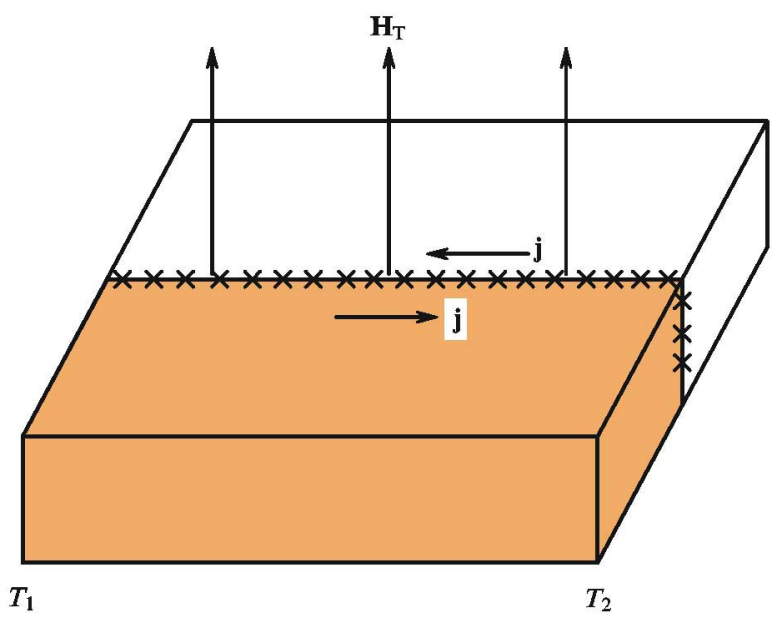

FIG. 8. (Color in online edition) Plates of two different superconductors fused (soldered) together, whose juncture is an alloy, in the presence of a temperature gradient. Thermoelectric current $\mathbf{j}$ is detectable via the magnetic field $\mathbf{H}$, which is perpendicular to the junction line. According to Ginsburg, 1944b.

$\nabla \mu / e$; in what follows I ignore this feature.

An important point is that the thermoelectric current $\mathbf{j}_{\mathrm{n}}$ exists in the uniform case in the superconducting state [Fig. 7(b)], but the field $H=0$. When the superconductor is nonuniform or anisotropic, the currents $\mathbf{j}_{\mathrm{s}}$ and $\mathbf{j}_{\mathrm{n}}$ do not in general compensate each other completely, and an observable thermoelectric magnetic field emerges, which was noted in Ginsburg (1944b). In days of old (60 years ago!), as noted above, alloys were regarded with some suspicion, and it was even unclear whether the London equation could be applied to alloys. That is why I restricted myself to a brief consideration of a bimetallic plate (say, of two different superconductors fused or soldered together, whose juncture is the alloy) in the presence of a temperature gradient [see also Chap. 16 in Ginzburg (1946b) and see Ginzburg and Zharkov (1978)]. In this case, because the parameter $\Lambda$ depends on the coordinates (evidently, the $\Lambda$ parameter is different for different metals), along the junction line there emerges an uncompensated current $\mathbf{j}$ and hence the magnetic field $\mathbf{H}$, which is perpendicular to the plate and the junction line (Fig. 8). Considered in greater detail in Ginsburg (1944b) and Ginzburg (1946b) was the case of an anisotropic superconductor. To this end, the London equations were generalized in a rather trivial way by replacing the scalar $\Lambda$ with the tensor $\Lambda_{i k}$ (for isotropic and cubic metals, $\Lambda_{i k}=\Lambda \delta_{i k}$ ). When the temperature gradient $\nabla T$ in a plate-shaped noncubic superconducting crystal is not directed along the symmetry axis, there emerges a current $\mathbf{j}$ flowing around the plate and a magnetic field $\mathbf{H}_{\mathrm{T}}$ transverse to the plate and proportional to $(\nabla T)^{2}$. In principle, this field is not difficult to observe with modern techniques. This is an interesting effect, which in addition makes it possible to measure the thermoelectric coefficient $b_{\mathrm{n}}(T)$ or, more precisely, the components of its generalization tensor $b_{\mathrm{n}, i k}(T)$. More than 30 years ago I managed to convince W. Fairbank to stage the corresponding experiment, and its results remain, as 


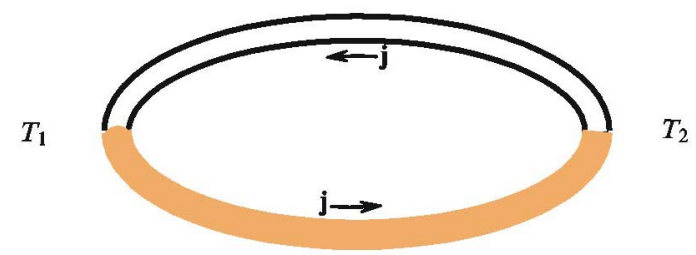

a)

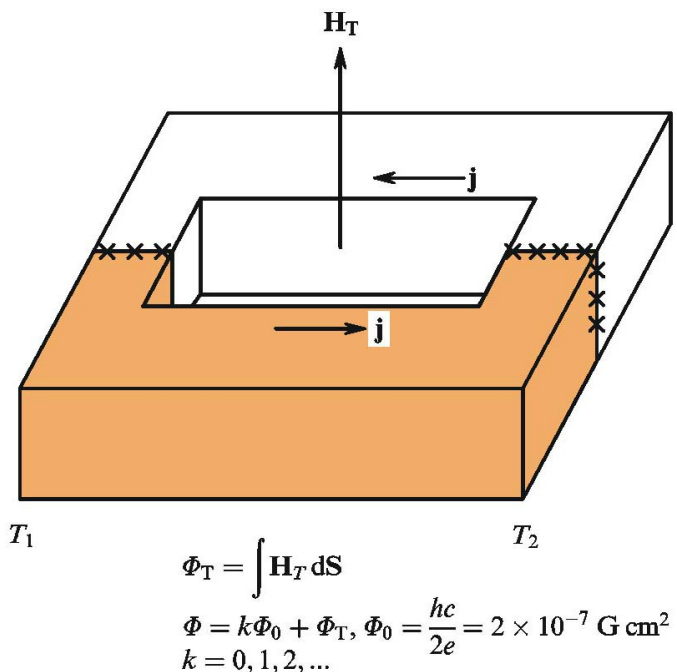

FIG. 9. (Color in online edition) Thermoelectric current in isotropic superconductors: (a) conventional thermoelectric current; (b) "circuit" of two superconducting metals, producing magnetic flux through the opening.

far as I know, the only ones on this subject (Selzer and Fairbank, 1974). Unfortunately, this work did not make things clear (Ginzburg and Zharkov, 1974, 1978). I am amazed by the fact that nobody has taken an interest in this question even after the fabrication of strongly anisotropic high-temperature superconductors. Evidently, such is the force of fashion in science, too.

True, a certain interest was attracted precisely by the isotropic superconductors, as applied to a more or less conventional thermoelectric current [Fig. 9(a)]. This circuit is equivalent to the "circuit" of Fig. 9(b). For this circuit it is easy to show (Gal'perin et al., 1974; Garland and Van Harlingen, 1974) (the derivation is also given in Ginzburg and Zharkov, 1978) that the magnetic flux $\Phi$ $=\int \mathbf{H d} \mathbf{S}$ through the opening is

$$
\begin{aligned}
& \Phi=k \Phi_{0}+\Phi_{\mathrm{T}}, \quad \Phi_{\mathrm{T}}=\frac{4 \pi}{c} \int_{T_{1}}^{T_{2}}\left(b_{\mathrm{n}, \mathrm{II}} \delta_{\mathrm{II}}^{2}-b_{\mathrm{n}, \mathrm{I}} \delta_{\mathrm{I}}^{2}\right) d T, \\
& \Phi_{0}=\frac{h c}{2 e}=2 \times 10^{-7} \mathrm{G} \mathrm{cm}^{2}, \quad k=0,1,2,3 \ldots
\end{aligned}
$$

Here, the indices I and II refer to the superconducting metals I and II, $\delta_{\mathrm{I}}$ and $\delta_{\mathrm{II}}$ are the field penetration depths for these metals, $b_{\mathrm{n}, \mathrm{I}}$ and $b_{\mathrm{n}, \mathrm{II}}$ are the corresponding coefficients $b_{n}(T)$ in Eq. (18), and $\Phi_{0}$ is the so-called flux quantum. The configuration in Fig. 9(b) is essentially equivalent to the bimetallic plate in Fig. 8 with $k=0$, i.e., without an opening. Unfortunately, I did not recognize this at the time (i.e., in Ginsburg, 1944b; Ginzburg, 1946b).

If we assume for simplicity that $\left(b_{\mathrm{n}} \delta^{2}\right)_{\mathrm{II}} \gg\left(b_{\mathrm{n}} \delta^{2}\right)_{\mathrm{I}}$ and $\delta_{\mathrm{II}}^{2}=\delta_{\mathrm{II}}^{2}(0)\left(1-T / T_{\mathrm{c}, \mathrm{II}}\right)^{-1}$, from expression (21) we obtain

$$
\Phi_{\mathrm{T}}=\frac{4 \pi}{c} b_{\mathrm{n}, \mathrm{II}}\left(T_{c}\right) \delta_{\mathrm{II}}^{2}(0) T_{\mathrm{c}} \ln \left(\frac{T_{\mathrm{c}}-T_{1}}{T_{\mathrm{c}}-T_{2}}\right) .
$$

If we substitute the known values $b_{\mathrm{n}}\left(T_{\mathrm{c}}\right)$ and $\delta(0)$ for $\ln \left(T_{\mathrm{c}}-T_{1}\right) /\left(T_{\mathrm{c}}-T_{2}\right) \sim 1$ in expression (22) we arrive at an estimate $\Phi_{\mathrm{T}} \sim 10^{-2} \Phi_{0}$. This flux is easy to measure, which was done in several papers (see Ginzburg, 1997, 1998; Ginzburg and Zharkov, 1978; and references therein). However, the flux $\Phi_{\mathrm{T}}$ observed in some more complex configuration of the superconducting circuit was found to be orders of magnitude higher than the flux given by expressions (21) and (22) and to possess a different temperature dependence (Van Harlingen, 1982). The reason for this result has not been elucidated, and different assumptions have been made on that score (Arutyunyan et al., 1997a, 1997b; Galperin et al., 2002); see also other references in Ginzburg (1997, 1998).

It is also pertinent to note that Eq. (21) and the ensuing formula (22) are obtained under the assumption that the equality $\mathbf{j}=\mathbf{j}_{\mathrm{s}}+\mathbf{j}_{\mathrm{n}}=0$ is fulfilled throughout the circuit depth (the current flows only near the surface). Meanwhile, as $T_{\mathrm{c}}$ is approached, the field penetration depth $\delta$ increases; as $T \rightarrow T_{\mathrm{c}}$, the depth $\delta \rightarrow \infty$ and the current density $\mathbf{j}_{\mathrm{n}}$ tends to the thermoelectric current density in the normal state, i.e., for $T>T_{\mathrm{c}}$. In these conditions, a more detailed analysis is required to include the charge imbalance effect. This interesting question has not been investigated (for more details, see Ginzburg, 1997, 1998).

The aforesaid is not the whole story. Even in the simplest case of a uniform superconductor, the existence of a temperature gradient [see Fig. 7(b)] affects the thermal conduction: since $\mathbf{j}_{\mathrm{n}} \neq 0$, there is bound to be an additional (convective) heat flux $\mathbf{q}_{\mathrm{c}}=-\varkappa_{c} \nabla T$ similar to that occurring in a superfluid liquid. This was noted even in Ginsburg (1944b) and was, in fact, the initial idea in this work.

The total heat flux in the superconducting state $\mathbf{q}$ $=-x \nabla T, x=x_{\mathrm{ph}}+x_{\mathrm{e}}+x_{\mathrm{c}}$ where $x_{p h}$ is the thermal conductivity coefficient related to the lattice (phonons), $x_{\mathrm{e}}$ is the electron contribution in the absence of convection (circulation), i.e., subject to the condition $\mathbf{j}_{\mathrm{n}}=0$, and, as already noted, $x_{\mathrm{c}}$ is the contribution of circulation. As is generally known, the thermal conductivity coefficient in the normal state is, by definition, measured for $\mathbf{j}=0$, and it is valid to say that $\varkappa_{\mathrm{c}}=0 .{ }^{9}$ When estimating the $x_{\mathrm{c}}$ coefficient, I, like others, got tangled up, and now I will restrict myself to a reference to paper (Ginzburg, 1997, 1998) and a remark that in ordinary (not hightemperature) superconductors supposedly $x_{\mathrm{c}} \ll x_{\mathrm{e}}$. The

\footnotetext{
${ }^{9}$ It is another matter that, for instance, a semiconductor subjected to the condition $\mathbf{j}=0$ in the presence of electron and hole conduction can simultaneously carry electron $\mathbf{j}_{\mathrm{c}}$ and hole $\mathbf{j}_{h}=-\mathbf{j}_{\mathrm{e}}$ currents; we ignore these possibilities.
} 
role of $x_{\mathrm{c}}$ in high-temperature superconductors is unclear to me. Most important of all, it is not clear how to extract $\varkappa_{\mathrm{c}}$, even if it were possible to determine separately $\varkappa_{\mathrm{ph}}$ and $\varkappa_{\mathrm{e} \text {,tot }}=\varkappa_{\mathrm{e}}+\varkappa_{\mathrm{c}}$ [the total thermal conductivity coefficient $x$ is measured directly; on the separation of $x_{\mathrm{ph}}$ from $x_{\mathrm{e} \text {,tot }}$, see Ginzburg $\left.(1997,1998)\right]$.

I can say no more here about thermoelectric effects in the superconducting state. My aim is to draw attention to this range of questions, which came under the scrutiny of science back in 1927 (see Meissner, 1927, as well as Shoenberg, 1965) and under mine in 1944 (Ginsburg, 1944b), but which remains largely unclear to date. This is so in spite of a multitude of papers concerned with superconductivity.

\section{SUPERFLUIDITY RESEARCH}

The $\Psi$ theory of superfluidity

Superconductivity is, if you please, the superfluidity of a charged liquid or, equivalently, superfluidity is the superconductivity of a noncharged liquid. It is therefore natural that the investigations of both effects have been interrelated. My first work in this area (Ginsburg, 1943), concerned with light scattering in helium II, was already mentioned in Sec. II. By the way, there is good reason to revert to this question in light of modern understanding of the fluctuations near the $\lambda$ point. Several other papers were dealt with in Ginzburg (1997, 1998); here, I will consider only the $\Psi$ theory of superfluidity, albeit with one exception. Namely, I would like to mention a proposal made jointly with A. A. Sobyanin ${ }^{10}$ and partly with G. F. Zharkov (Ginzburg and Sobyanin, 1983; Ginzburg et al., 1974, 1981), and then mention the study of the feasibility of observing the thermomechanical circulation effect in a superfluid liquid.

In an annular vessel filled with a superfluid liquid (in this case, helium II), which has two different "bottlenecks" (for instance, narrow capillaries), under a temperature gradient there is bound to emerge a circulation-a superfluid flow engulfing the entire vessel (Fig. 10). By the way, we made the inference about the existence of this effect (Ginzburg et al., 1974, 1981) on the basis of analogy with the thermoelectric effect in a superconducting circuit. As to the inference about the existence of thermoelectric current in a superconducting circuit, I made it (Ginsburg, 1944b) at the time on the strength of analogy with the behavior of helium II under a temperature gradient. The above thermocirculation effect in helium II has been observed (Gamtsemlidze and Mirzoeva, 1980, 1983) and discussed (Ginzburg and Sobyanin, 1983), and, in my view, interesting possibilities were pointed out for future research (Ginzburg and Sobyanin, 1983). However, nobody, as far as I know, has

\footnotetext{
${ }^{10}$ The talented theoretical physicist and public figure Aleksandr Sobyanin (Fig. 11) died prematurely at the age of 54 in 1997.
}

$$
\begin{gathered}
\oint \mathbf{v}_{\mathbf{s}} \mathrm{d} \mathbf{l}=2 \pi \frac{\hbar}{m} k, \quad k=0, \pm 1, \pm 2, \ldots \\
\frac{2 \pi \hbar}{m_{4} \mathrm{He}} \approx 10^{-3} \mathrm{~cm}^{2} \mathrm{~s}^{-1}
\end{gathered}
$$

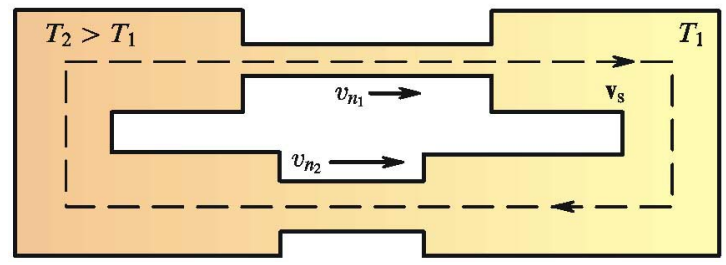

FIG. 10. (Color in online edition) Proposal for an experiment to produce superfluid flow. According to Ginzburg and Sobyanin, 1983.

taken an interest in this question during the past 20 years.

After the development of the $\Psi$ theory of superconductivity (Ginzburg and Landau, 1950), the transfer of something similar to the case of superfluidity appeared to be rather obvious. Even before this time (see, for instance, Ginsburg, 1944a), I was concerned about the behavior of helium II near the $\lambda$ point, and the question of the boundary condition for the superfluid component velocity $\mathbf{v}_{\mathrm{s}}$ was obscure. By the way, Landau, the originator of the theory of phase transitions and superfluidity, for some reason was never concerned with this subject, as far as I know. In the Landau theory of superfluidity (Landau, 1941), the velocity $\mathbf{v}_{\mathrm{s}}$ along the wall (unlike the normal-component velocity $\mathbf{v}_{\mathrm{n}}$ ) does not vanish at the wall: there is some kind of discontinuity. But in this case, it seemed to me, this discontinuity was bound to be related to some surface energy $\sigma_{\mathrm{s}}$ (Ginsburg, 1955b). However, dedicated experiments (Gamtsemlidze, 1958) showed that the $\sigma_{\mathrm{s}}$ energy is nonexistent or, in any case, is many orders of magnitude lower than the expected energy (Ginsburg, 1955b). I saw a way out in the assumption that the density of the superfluid component at the wall $\rho_{\mathrm{s}}(0)$ is zero. Then, the superfluid component flux $\mathbf{j}_{\mathrm{s}}=\rho_{\mathrm{s}} \mathbf{v}_{\mathrm{s}}$ at the wall vanishes despite the fact that $\mathbf{v}_{\mathrm{s}}$

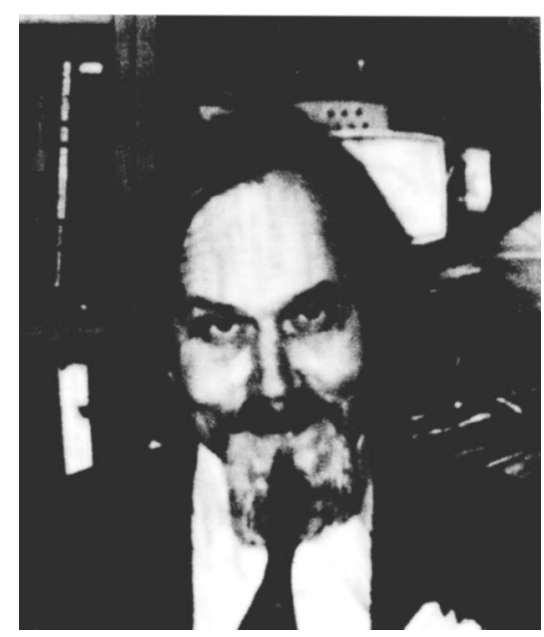

FIG. 11. A. A. Sobyanin, 1943-1997. 


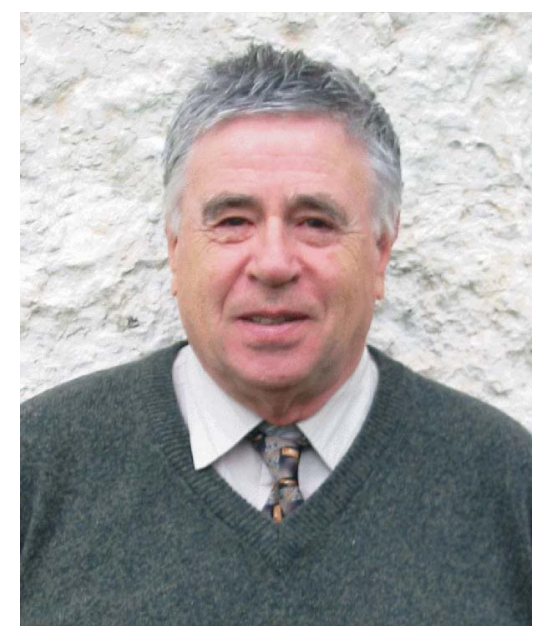

FIG. 12. (Color in online edition) L. P. Pitaevskii.

has a discontinuity at the wall. In the $\Psi$ theory of superfluidity, evidently,

$$
\rho_{\mathrm{s}}=m|\Psi|^{2}
$$

where it may be assumed that $m=m_{\mathrm{He}}$ is the mass of a helium atom (we imply the superfluidity of helium II) and, in view of the foregoing, the boundary condition at the wall is

$$
\Psi(0)=0,
$$

instead of the condition (3) for superconductors. At this stage, as far as I remember, it turned out that L. P. Pitaevskii (see Fig. 12) had independently taken up the $\Psi$ theory of superfluidity and, naturally, we combined efforts. As a result, an article (Ginzburg and Pitaevskii, 1958) emerged; I speak of the $\Psi$ theory of superfluidity constructed in that work as "initial" because I consider below the "generalized" $\Psi$ theory of superfluidity elaborated together with Sobyanin (Ginzburg and Sobyanin, 1976, 1982, 1987a, 1988; see also several other references in Ginzburg, 1997, 1998).

The initial $\Psi$ theory of superfluidity (Ginzburg and Pitaevskii, 1958) is quite similar to the $\Psi$ theory of superconductivity (Ginzburg and Landau, 1950), of course, with the use of the boundary condition (24) and in the absence of electric charge. In this case, the scalar complex function $\Psi=|\Psi| \exp (i \varphi)$ obeys the equation

$$
-\frac{\hbar^{2}}{2 m} \Delta \Psi+\alpha(T) \Psi+\beta_{\lambda}|\Psi|^{2} \Psi=0
$$

and

$$
\mathbf{j}_{\mathrm{s}}=\rho_{\mathrm{S}} \mathbf{v}_{\mathrm{s}}=-\frac{i \hbar}{2}\left(\Psi^{*} \nabla \Psi-\Psi \nabla \Psi^{*}\right)=\hbar|\Psi|^{2} \nabla \varphi,
$$

i.e., $\mathbf{v}_{s}=(\hbar / m) \nabla \varphi$, with $m=m_{\mathrm{He}}$ irrespective of how $\Psi$ is normalized (see Ginzburg and Pitaevskii, 1958; Ginzburg, 1997, 1998).

Furthermore, the correlation length $\xi$ denoted as $l$ in Ginzburg and Pitaevskii (1958) is

$$
\xi(T)=\frac{\hbar}{\sqrt{2 m \mid \alpha}}=\xi(0) \tau^{-1 / 2}, \quad \tau=\frac{T_{\lambda}-T}{T_{\lambda}},
$$

where $T_{\lambda}$ is the temperature of the $\lambda$ point. The estimate of Ginzburg and Pitaevskii (1958), based on experimental data, for ${ }^{4} \mathrm{He}$, i.e., for helium II, leads to a value $\xi(0) \sim 3 \times 10^{-8} \mathrm{~cm}$. At the same time, the $\Psi$ theory is applicable only when the macroscopic $\Psi$ function varies only slightly over atomic-scale distances. Hence there follows the condition $\xi(T) \gg a \sim 3 \times 10^{-8} \mathrm{~cm}$ (here $a$ is the average interatomic distance in liquid helium). The $\Psi$ theory can therefore be adequate only near the $\lambda$ point (for $\tau \ll 1)$, say, for $\left(T_{\lambda}-T\right)<(0.1-0.2) \mathrm{K}$. A similar condition also applies in the case of the $\Psi$ theory of superconductivity, which is also appropriate, generally speaking, only near $T_{\mathrm{c}}$. It is of prime importance that the Landau theory of phase transitions, which is a meanfield theory, for superconductors (i.e., the $\Psi$-theory of superconductivity) also be correct in the immediate vicinity of $T_{\mathrm{c}}$. This is due to the relatively large value of $\xi(0)$ in superconductors [the length $\xi(0)$ is on the order of the dimension of the Cooper pairs, i.e., in ordinary superconductors is on the order of, say, $\left.10^{-5} \mathrm{~cm}\right]$. The point is that the temperature range near $T_{\mathrm{c}}\left(\right.$ or $T_{\lambda}$ ), in which fluctuations are already large and the mean-field approximation is inappropriate, is proportional to $[\xi(0)]^{-6}$ (see Ginzburg, 1997, 1998, and references therein, particularly Ginzburg, 1960). In helium II, the fluctuations near $T_{\lambda}$ are relatively strong due to the smallness of $\xi(0)$, and the $\Psi$ theory (Ginzburg and Pitaevskii, 1958) can be used only for $\left(T_{\lambda}-T\right) \gg 10^{-3} \mathrm{~K}$ (Ginzburg, 1997, 1998). Meanwhile, the temperature range significantly closer to $T_{\lambda}$ is of special interest. That the mean-field theory is inapplicable in the region of the $\lambda$ transition in ${ }^{4} \mathrm{He}$ is testified to by the very existence of the $\lambda$ singularity in the temperature dependence of the heat capacity. This circumstance might not, at least on the face of it, be related to the temperature dependence of the density $\rho_{\mathrm{s}}(T)$, which was proportional to $|\Psi|^{2}$ [see Eq. (23)]. That is why in 1957, when Pitaevskii and I carried out the work for our 1958 article, we did not see the drawbacks to our theory right away. However, this became clear somewhat later, when it was found out that in helium II to a good approximation

$$
\rho_{\mathrm{s}}(\tau)=\rho_{\mathrm{s} 0} \tau^{\zeta}, \quad \zeta=\frac{2}{3} .
$$

In the mean-field theory,

$$
\zeta=1 .
$$

In experiment, by the way, the index $\zeta$ is not exactly equal to $\frac{2}{3}$ but is very close to it. For instance, according to Golder et al. (1992), $\zeta=0.6705 \pm 0.0006$.

Therefore the initial $\Psi$ theory of superfluidity (Ginzburg and Pitaevskii, 1958) is poorly applicable to liquid ${ }^{4} \mathrm{He}$ in a quantitative sense. At the same time, several results based on it were of significance for helium II in a qualitative sense, in particular, the density distribution 
$\rho_{s}(z)$ near the solid wall and in films with a thickness $d$ in relation to this thickness. Also solved were the problems of velocity $\mathbf{v}_{\mathrm{s}}$ circulation about a vortex filament at the axis of which $\Psi=0$, of the energy of this filament, and of the surface energy at the interface between helium II and the solid wall. Of course, liquid ${ }^{4} \mathrm{He}$ is not the only existing superfluid liquid. Such a liquid is also encountered in ${ }^{3} \mathrm{He}-{ }^{4} \mathrm{He}$ solutions, liquid ${ }^{3} \mathrm{He}$, neutron stars, and maybe in other cases. In such cases, however, the $\Psi$ function may prove to be no longer scalar but, on the other hand, the length $\xi(0)$ is relatively large [in liquid ${ }^{3} \mathrm{He}$, for instance, $\left.\xi(0) \sim 10^{-5} \mathrm{~cm}\right]$, and the fluctuation region is rather small. Finally, the theory of Ginzburg and Pitaevskii (1958) played, so far as I can judge, a significant role in the construction and elaboration of the Gross-Pitaevskii theory, which is widely used in the investigation of Bose-Einstein condensation (see Pitaevskii and Stringari, 2003).

Liquid ${ }^{4} \mathrm{He}$, i.e., helium II, has always occupied and still occupies the leading position in the physics of superfluidity, both historically and regarding the scale of investigations. The Landau theory (Landau, 1941), which describes its behavior, is primarily macroscopic or, if you like, quasimacroscopic. But it does not provide answers to several questions, particularly near the $\lambda$ point. At the same time, a microtheory of the BCS type for superconductors does not exist for helium II. On the other hand, helium II near the $\lambda$ point is interesting from various viewpoints, in particular, in the investigation of twoliquid hydrodynamics near the $\lambda$ point, in the modeling of some cosmological situations (Zurek, 1996), etc. It is likely that the initial $\Psi$ theory of superfluidity (Ginzburg and Pitaevskii, 1958; Pitaevskii, 1958) can be used to some extent for the solution of these problems, though with the above significant limitation arising from the inapplicability of the mean-field approximation, i.e., from the neglect of fluctuations. The generalized $\Psi$ theory of superfluidity (Ginzburg and Sobyanin, 1976, 1982, 1987a, 1988) was intended to eliminate these drawbacks. It is based on a semiempirical generalization of the Landau theory of phase transitions (see, for instance, Mamaladze, 1967, 1968). In the Landau theory of phase transitions and, in particular, in the $\Psi$ theory of superconductivity, i.e., when the $\Psi$ function is selected as the order parameter, the free energy density of the ordered phase near the transition point $T_{\lambda}$ is written in the form

$$
F_{\mathrm{II}}=F_{\mathrm{I}}+\alpha|\Psi|^{2}+\frac{\beta}{2}|\Psi|^{4}+\frac{\gamma}{6}|\Psi|^{6}
$$

away from the tricritical point, it being safe to assume that

$$
\begin{aligned}
& \alpha=\alpha_{\lambda}^{\prime}\left(T-T_{\lambda}\right)=-a_{0} \tau, \quad \beta=\beta_{\lambda}, \\
& \gamma=0, \quad \tau=\frac{T_{\lambda}-T}{T_{\lambda}} .
\end{aligned}
$$

In the generalized theory

$$
F_{\mathrm{II}}=F_{\mathrm{I}}-a_{0} \tau|\tau|^{1 / 3}|\Psi|^{2}+\frac{b_{0}}{2} \tau^{2 / 3}|\Psi|^{4}+\frac{g_{0}}{3}|\Psi|^{6} .
$$

When selecting Eq. (32), one finds for small $|\Psi|^{2}$ in equilibrium $\left|\Psi_{0}\right|^{2}=-\alpha / \beta=\left(a_{0} / b_{0}\right) \tau^{2 / 3}$, i.e., there occurs a temperature dependence that agrees with the observed one [see Ginsburg (1952b)]. Clearly Eq. (32) is selected for precisely the attainment of this goal.

The generalized $\Psi$ theory of superfluidity (Ginzburg and Sobyanin, 1976, 1982, 1987a, 1988) formally differs from the initial theory (Ginzburg and Pitaevskii, 1958; Pitaevskii, 1958) just by the replacement of Eqs. (30) and (31) with Eq. (32). Several expressions and inferences were derived on this basis. For instance, for a thin film of helium II of thickness $d$, the $\lambda$-transition temperature is

$$
T_{\lambda}(d)=T_{\lambda}-2.53 \times 10^{-11}\left(\frac{3+M}{M}\right) d^{-3 / 2} \mathrm{~K},
$$

where $T_{\lambda}=T_{\lambda}(\infty)$ is the $\lambda$-transition temperature in massive helium (as is well known, $T_{\lambda}=2.17 \mathrm{~K}$ ) and $M$ is the parameter of the theory proportional to the $g_{0}$ coefficient in Eq. (32). When $M<1$, the $\lambda$ transition is of the second kind (by comparison with experiment, only a crude estimate was obtained for helium II: $M=0.5 \pm 0.3$ ). By the way, if we consider a cylindrical capillary of diameter $d$ instead of a plane film, the coefficient 2.53 in Eq. (33) should be replaced with 4.76. Quite a number of other expressions were also derived (Ginzburg and Sobyanin, 1976, 1982, 1987a, 1987b, 1988).

Unfortunately, the generalized $\Psi$ theory of superfluidity has not come to the attention of either experimenters or theorists. True, some pessimistic judgments regarding it were expressed in the literature (they were mentioned in Ginzburg, 1997, 1998). Sobyanin and I abandoned superfluidity research during the period of rapid change in the USSR and Russia that set in after 1985-1988. Only in Ginzburg $(1997,1998)$ did I review our work.

Undeniably the generalized $\Psi$ theory of superfluidity is not a lofty $a b$ initio theory. At the same time, its simplicity (at least in comparison with other known methods) suggests that both it and the initial version of the theory can still yield much in the study of superfluidity. In any case, the opposite opinion is not substantiated at all. This section of the lecture has been written precisely with the aim of attracting the attention of physicists engaged in related areas to the $\Psi$ theory of superfluidity. It may well be that the lack of attention is a mistaken impression on my part. It is conceivable, on the contrary, that I am in error myself, though.

\section{THE "PHYSICAL MINIMUM"-WHAT PROBLEMS OF PHYSICS AND ASTROPHYSICS SEEM NOW TO BE ESPECIALLY IMPORTANT AND INTERESTING AT THE BEGINNING OF THE 21ST CENTURY?}

I have encountered the viewpoint that my work in the area of superconductivity and superfluidity belongs to the remote past. There is no question that the work of 


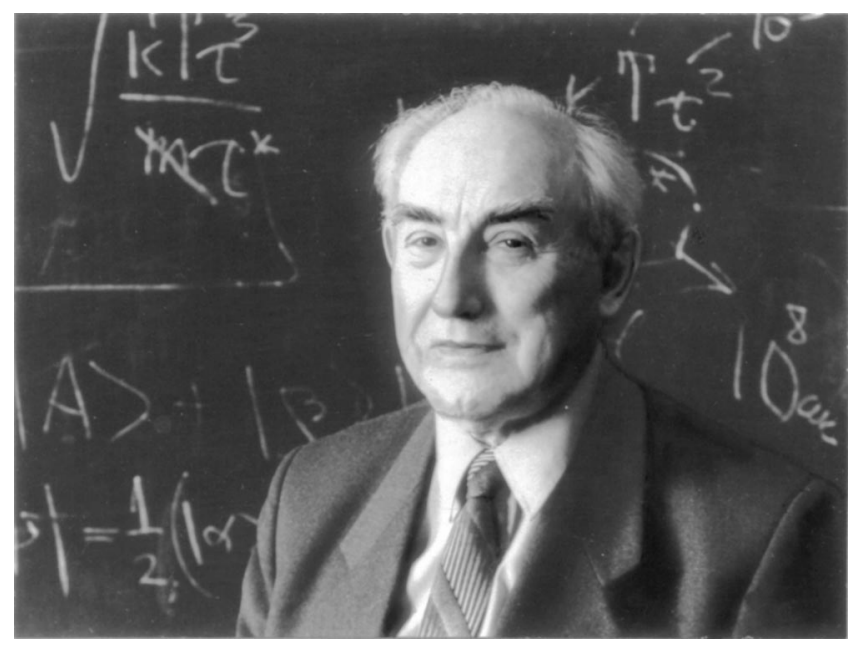

FIG. 13. The author, V. L. Ginzburg, at the weekly seminar he held at the Fizicheskii Institut Academii Nauk (FIAN, the P. N. Lebedev Physical Institute of the Academy of Sciences) over many years. This seminar on theoretical physics, which became rather famous for its lively exchange of information and ideas, was as enjoyable for the teacher as for the participants. By the time of its last session on November 21, 2001, it had met 1700 times.

Ginzburg and Landau (Ginzburg and Landau, 1950) performed back in 1950 stands out. But, as is clear from the foregoing (Ginzburg, 1997, 1998), I have been occupied with this field of physics since 1943 until the present time (see Fig. 13). In this case, it seems to me, several questions have been posed and problems addressed which have not been solved and which deserve attention. Of course, at present the most urgent problems in the area of superconductivity are the elucidation of the mechanism and several features of high-temperature superconductivity and the development of room-temperature superconductivity. More precisely, what is wanted in the latter case is to identify the potentialities and formation conditions of room-temperature superconductors. I am keenly aware that I will not be able to accomplish anything in the last two directions. I would like only to witness as many new findings as possible.

That is why in recent years I have been placing progressively stronger emphasis, as far as physics is concerned, on an educational program, which I conventionally call the "physical minimum." As far as I know, many young scientists attend Nobel Lectures, and therefore I decided to enlarge on this "physical minimum." I believe that this will be of greater interest to young people than to hear what was going on before they were born.

Physics has developed rapidly and fruitfully, especially in the past century. Its face has changed radically even within a human life span. I myself was already 16 when the neutron and positron were discovered in 1932. And what would modern physics be without neutrons and positrons? As a result of so rapid a development, physics and the adjacent realms of study (for instance, astronomy) have enormously expanded, both as regards their basic concerns and the body of information. In the recent past it was possible to be guided by the require- ment "to know something about everything and to know everything about something" (say, in physics), but now, it seems to me, this is no longer possible. At the same time, I am startled and dispirited when young physicists (and sometimes not so young ones) restrict themselves to the knowledge in "their" area and are not informed, even in a general way, about the state of physics as a whole and its "hottest" areas.

This situation cannot be justified by alleging the absence of a pivot (keystone) in contemporary physics or its boundlessness. Quite the contrary. Physics does have its pivot, which is represented by fundamental concepts and laws formulated in theoretical physics. It is possible, on the basis of theoretical physics studied during one's student days, to understand all modern physics or, more precisely, to understand how matters stand everywhere in physics and be aware of the research situation. Every physicist (naturally, this equally applies to other sciences, but I restrict myself to physicists here) should simultaneously know, apart from theoretical physics, a wealth of facts from different branches of physics and be familiar with the newest notable accomplishments.

We in Russia like to quote a certain Koz'ma Prutkov, a fictitious character, who said pompously, in particular, that "there is no way of comprehending the incomprehensible." So, one has to choose something. And so I took this path: I have addressed the question, "What should a physicist know something about?" by making a list of the top problems of the day. Any such list is admittedly subjective. It is also clear that it will vary with time. Lastly, it is clear that subjects not included in the list can in no way be regarded as unimportant or uninteresting. It is simply that many of them presently seem less pressing to me (or to the authors of other similar lists). Those who know interesting subjects beyond the list have no reason to be offended and should only supplement or make their own versions of it. I only suggest some enumeration of the questions that, in my view, every physicist should have at least a superficial idea of. This is not as difficult as it might seem at first glance. The time to be spent for this purpose is, I believe, no longer than the time a good student spends preparing for an examination, say, on electrodynamics.

Acquaintance with all subjects included in this list is what I call the "physical minimum." Of course, this minimum is the echo of the "theoretical minimum" proposed by Landau in the 1930s. The corresponding volume of the "Course of Theoretical Physics" by L. D. Landau and E. M. Lifshitz ranks, in my view, highest among the many excellent textbooks on electrodynamics. But a beginner needs help to get acquainted with the "physical minimum." Working out this list, as well as commenting on it, is the first step towards this goal. In 1995, in the Russian edition of my book Reflections on the Problems and Personalities of 20th Century Physics, I managed to work out a rather detailed commentary. But in the English translation (Ginzburg, 2001) some parts were already out of date, which I failed to compensate for in full measure. Inserted at the beginning of the book Ginzburg (2003) is an article also concerned with the 
"physical minimum." Several additional remarks were introduced in the English translation of this book, which will hopefully be published soon. Should the proposal for a "physical minimum" meet with support, perhaps new books on this subject will appear. Unfortunately, I cannot set myself to this task.

So, what is the "physical minimum" that a physicist should know about? Here is my list for the beginning of the 21st century:

1. Controlled nuclear fusion.

2. High-temperature and room-temperature superconductivity.

3. Metallic hydrogen. Other exotic substances.

4. Two-dimensional electron liquid (the anomalous Hall effect and other effects).

5. Some questions of solid-state physics (heterostructures in semiconductors, quantum wells and dots, metal-dielectric transitions, charge- and spindensity waves, mesoscopics).

6. Second-order and related phase transitions. Some examples of such transitions. Cooling (in particular, laser cooling) to superlow temperatures. BoseEinstein condensation in gases.

7. Surface physics. Clusters.

8. Liquid crystals. Ferroelectrics. Ferrotoroics.

9. Fullerenes. Nanotubes.

10. The behavior of matter in superstrong magnetic fields.

11. Nonlinear physics. Turbulence. Solitons. Chaos. Strange attractors.

12. X-ray lasers, gamma-ray lasers, superhigh-power lasers.

13. Superheavy elements. Exotic nuclei.

14. Mass spectrum. Quarks and gluons. Quantum chromodynamics. Quark-gluon plasma.

15. Unified theory of weak and electromagnetic interactions. $W^{ \pm}$and $Z^{0}$ bosons. Leptons.

16. Standard Model. Grand unification. Superunification. Proton decay. Neutrino mass. Magnetic monopoles.

17. Fundamental length. Particle interaction at high and superhigh energies. Colliders.

18. Nonconservation of CP invariance.

19. Nonlinear phenomena in vacuum and in superstrong magnetic fields. Phase transitions in a vacuum.

20. Strings. M theory.

21. Experimental verification of the general theory of relativity.

22. Gravitational waves and their detection.

23. The cosmological problem. Inflation. The $\Lambda$ term and "quintessence." Relationship between cosmology and high-energy physics.

24. Neutron stars and pulsars. Supernova stars.

25. Black holes. Cosmic strings(?).

26. Quasars and galactic nuclei. Formation of galaxies.

27. The problem of dark matter (hidden mass) and its detection.

28. The origin of superhigh-energy cosmic rays.

29. Gamma-ray bursts. Hypernovae.

30. Neutrino physics and astronomy. Neutrino oscillations.

The singling out of 30 particular problems (as constituting the minimum) is of course absolutely subjective. Moreover, some of them might be subdivided. In my first such list, published in 1971, there were 17 problems (Ginzburg, 1971). Subsequently their number grew (for details, see Ginzburg, 2003). Some worthy new subjects that might be added would be in the fields of quantum computers and advances in optics. But I cannot do this with adequate comprehension.

Any such list undoubtedly will evolve with time, as it reflects the development of physics. In my first list (Ginzburg, 1971) quarks were given only three lines in the enumeration of the attempts to explain the mass spectrum. This did not testify to my perspicacity. However, at that time (in 1970) quarks were only five or six years old (I mean the age of the corresponding hypothesis), and the fate of the concept of the quark was indeed vague. Now the situation is of course quite different. True, the heaviest $t$ quark was discovered only in 1994 (its mass, according to the data of 1999, is $m_{\mathrm{t}}$ $=176 \pm 6 \mathrm{GeV}$ ). That early list (Ginzburg, 1971) naturally contains no fullerenes, which were discovered in 1985, and no gamma-ray bursts (the first report of their discovery was published in 1973). High-temperature superconductors were synthesized in 1986-1987, but do appear in the list (Ginzburg, 1971) because this problem had been discussed since 1964 (as described in the previous sections of the lecture). Generally, much has been done in physics over the past 30 or 35 years, but, I believe, not very much that warrants a new addition to the list. In any case, the two earlier lists (Ginzburg, 1971, 2001), as well as that presented above, characterize to a certain extent the development and the state of physical and astronomical problems from 1970-1971 to the present day.

It should be added that three "great problems" of modern physics are also to be included in the "physics minimum," included in the sense that they should be singled out in some way and specially discussed, and their development should be reviewed. This is discussed at some length in the book About Science, Myself, and Others (Ginzburg, 2003). The "great problems" are, first, the increase in entropy, time irreversibility, and the "time arrow." Second is the problem of interpretation of nonrelativistic quantum mechanics and the possibility of 
learning something new even in the field of its applicability (I personally doubt this possibility but believe that one's eyes should remain open). And third is the question of the emergence of life, i.e., the feasibility of explaining the origin of life and thought on the basis of physics alone. On the face of it, how could it be otherwise? But until the questions are elucidated, one cannot be quite sure of anything. I think that the problem of the origin of life will unreservedly be solved only after "life in a test-tube" is created. Until then, this will be an open question.

One more concluding remark. In the past century, and even nowadays, one could encounter the opinion that in physics nearly everything had been done. There allegedly are only dim "cloudlets" in the sky or theory, which will soon be eliminated to give rise to the "theory of everything." I consider these views as some kind of blindness. The entire history of physics, as well as the state of present-day physics and, in particular, astrophysics, testifies to the opposite. In my view we are facing a boundless sea of unresolved problems.

It only remains for me to envy the younger members of the audience, who will witness a great many new, important, and interesting things.

\section{REFERENCES}

Abrikosov, A. A., 1957, Zh. Eksp. Teor. Fiz. 32, 1442 [Sov. Phys. JETP 5, 1174 (1957)].

Arutyunyan, R. M., V. L. Ginzburg, and G. F. Zharkov, 1997a, Zh. Eksp. Teor. Fiz. 111, 2175 [JETP 84, 1186 (1997)].

Arutyunyan, R. M., V. L. Ginzburg, and G. F. Zharkov, 1997b, Usp. Fiz. Nauk 167, 457 [Phys. Usp. 40, 435 (1997)].

Bardeen, J., 1956, in Kältenphysik, Handbuch der Physik Vol. 15, edited by S. von Flügge (Springer, Berlin), p. 274 [Translation: Fizika Nizkikh Temperatur, edited by A. I. Shal'nikov (IL, Moscow, 1959), p. 679].

Bardeen, J., L. N. Cooper, and J. R. Schrieffer, 1957, Phys. Rev. 108, 1175.

Bednorz, J. G., and K. A. Muller, 1986, Z. Phys. B: Condens. Matter 64, 189.

Buckel, W., 1972, Supraleitung (Physik, Weinheim, Bergster) [English translation: Superconductivity: Fundamentals and Applications (VCH, Weinheim, 1991); Russian translation: (Mir, Moscow, 1975)].

Burton, E. F., G. H. Smith, and J. O. Wilhelm, 1940, Phenomena at the Temperature of Liquid Helium, American Chemical Society Monograph Series, No. 83 (Reinhold, New York).

Cava, R. J., R. B. van Dover, B. Batlogg, and E. A. Rietman, 1987, Phys. Rev. Lett. 58, 408.

Cooper, L. N., 1956, Phys. Rev. 104, 1189.

Eliashberg, G. M., 1960a, Zh. Eksp. Teor. Fiz. 38, 966 [Sov. Phys. JETP 11, 696 (1960)].

Eliashberg, G. M., 1960b, Zh. Eksp. Teor. Fiz. 39, 1437 [Sov. Phys. JETP 12, 1000 (1961)].

Galperin, Y. M., V. L. Gurevich, V. I. Kozub, and A. L. Shelankov, 2002, Phys. Rev. B 65, 064531.

Gal' perin, Y. M., V. L. Gurevich, and V. N. Kozub, 1974, Zh.

Eksp. Teor. Fiz. 66, 1387 [Sov. Phys. JETP 39, 680 (1974)].

Gamtsemlidze, G. A., 1958, Zh. Eksp. Teor. Fiz. 34, 1434 [Sov.

Phys. JETP 7, 992 (1958)].

Gamtsemlidze, G. A., and M. I. Mirzoeva, 1980, Zh. Eksp.
Teor. Fiz. 79, 921 [Sov. Phys. JETP 52, 468 (1980)].

Gamtsemlidze, G. A., and M. I. Mirzoeva, 1983, Zh. Eksp.

Teor. Fiz. 84, 1725 [Sov. Phys. JETP 57, 1006 (1983)].

Garland, J. C., and D. J. Van Harlingen, 1974, Phys. Lett. 47A, 423.

Ginsburg, V. L., 1944a, Zh. Eksp. Teor. Fiz. 14, 134.

Ginsburg, V. L., 1944b, Zh. Eksp. Teor. Fiz. 14, 177 [J. Phys.

(USSR) 8, 148 (1944)].

Ginsburg, V. L., 1952a, Usp. Fiz. Nauk 48, 25.

Ginsburg, V. L., 1952b, Zh. Eksp. Teor. Fiz. 23, 236.

Ginsburg, V. L., 1955a, Zh. Eksp. Teor. Fiz. 29, 748 [Sov. Phys. JETP 2, 589 (1956)].

Ginsburg, V. L., 1955b, Zh. Eksp. Teor. Fiz. 29, 254 [Sov. Phys. JETP 2, 170 (1956)].

Ginzburg, V. L., 1943, Zh. Eksp. Teor. Fiz. 13, 243 [J. Phys. (USSR) 7, 305 (1943)].

Ginzburg, V. L., 1945, Zh. Eksp. Teor. Fiz. 15, 739 [J. Phys. (USSR) 10, 107 (1946)].

Ginzburg, V. L., 1946a, Zh. Eksp. Teor. Fiz. 16, 87 [J. Phys. (USSR) 9, 305 (1945)].

Ginzburg, V. L., 1946b, Sverkhprovodimost' Superconductivity (Izd. AN SSSR, Moscow-Leningrad).

Ginzburg, V. L., 1953, Fortschr. Phys. 1, 101.

Ginzburg, V. L., 1956a, Zh. Eksp. Teor. Fiz. 31, 541 [Sov. Phys. JETP 4, 594 (1957)].

Ginzburg, V. L., 1956b, Zh. Eksp. Teor. Fiz. 31, 202 [Sov. Phys. JETP 4, 153 (1957)].

Ginzburg, V. L., 1958, Zh. Eksp. Teor. Fiz. 34, 113 [Sov. Phys. JETP 7, 78 (1958)].

Ginzburg, V. L., 1959, Zh. Eksp. Teor. Fiz. 36, 1930 [Sov. Phys. JETP 9, 1372 (1959)].

Ginzburg, V. L., 1960 Fiz. Tverd. Tela 2, 2031 [Sov. Phys. Solid State 2, 1824 (1961)].

Ginzburg, V. L., 1962, Zh. Eksp. Teor. Fiz. 42, 299 [Sov. Phys. JETP 15, 207 (1962)].

Ginzburg, V. L., 1964a, Phys. Lett. 13, 101.

Ginzburg, V. L., 1964b, Zh. Eksp. Teor. Fiz. 47, 2318 [Sov. Phys. JETP 20, 1549 (1965)].

Ginzburg, V. L., 1971, Usp. Fiz. Nauk 103, 87 [Sov. Phys. Usp. 14, 21 (1971)].

Ginzburg, V. L., 1984, Energiya No. 9, 2.

Ginzburg, V. L., 1986, J. Supercond. 4, 327.

Ginzburg, V. L., 1989a, Phys. Scr., T T27, 76.

Ginzburg, V. L., 1989b, Prog. Low Temp. Phys. 12, 1.

Ginzburg, V. L., 1996, in From High-Temperature Superconductivity to Microminiature Refrigeration, edited by B. Cabrera, H. Gutfreund, and V. Kresin (Plenum, New York).

Ginzburg, V. L., 1997, Usp. Fiz. Nauk 167, 429 [Phys. Usp. 40, 407 (1997)].

Ginzburg, V. L., 1998, Usp. Fiz. Nauk 168, 363 [Phys. Usp. 41, 307 (1998)].

Ginzburg, V. L., 2001, The Physics of a Lifetime: Reflections on the Problems and Personalities of 20th Century Physics (Springer, Berlin) [this book is, for the most part, a translation of V. L. Ginzburg, O Fizike i Astrofizike (Byuro Kvantum, Moscow, 1995)].

Ginzburg, V. L., 2003, O Nauke, o Sebe i o Drugikh (About Science, Myself, and Others) (Fizmatlit, Moscow) (translation, IOP, Bristol, 2004).

Ginzburg, V. L., and D. A. Kirzhnits, 1964, Zh. Eksp. Teor. Fiz. 46, 397 [Sov. Phys. JETP 19, 269 (1964)].

Ginzburg, V. L., and D. A. Kirzhnits, 1967, Dokl. Akad. Nauk SSSR 176, 553 [Sov. Phys. Dokl. 12, 880 (1968)]. 
Ginzburg, V. L., and D. A. Kirzhnits, 1977, Eds., Problema Vysokotemperaturnoi Sverkhprovodimosti (The Problem of High-Temperature Superconductivity) (Nauka, Moscow), [Translation, High-Temperature Superconductivity (Consultants Bureau, New York, 1982)].

Ginzburg, V. L., and L. D. Landau, 1950, Zh. Eksp. Teor. Fiz. 20, 1064 [translation in L. D. Landau, Collected Papers (Pergamon, Oxford, 1965), p. 546].

Ginzburg, V. L., and E. G. Maksimov, 1992, Sverkhprovodimost': Fiz., Khim., Tekh. 5, 1543 [Supercond., Phys. Chem. Technol. 5, 1505 (1992)].

Ginzburg, V. L., and L. P. Pitaevskii, 1958, Zh. Eksp. Teor. Fiz. 34, 1240 [Sov. Phys. JETP 7, 858 (1958)].

Ginzburg, V. L., and A. A. Sobyanin, 1976, Usp. Fiz. Nauk 120, 153 [Sov. Phys. Usp. 19, 773 (1976)].

Ginzburg, V. L., and A. A. Sobyanin, 1982, J. Low Temp. Phys. 49, 507.

Ginzburg, V. L., and A. A. Sobyanin, 1983, Zh. Eksp. Teor. Fiz. 85, 1606 [Sov. Phys. JETP 58, 934 (1983)].

Ginzburg, V. L., and A. A. Sobyanin, 1987a, Jpn. J. Appl. Phys., Part 1, 26, 1785, Suppl. 26-3.

Ginzburg, V. L., and A. A. Sobyanin, 1987b, in Superconductivity, Superdiamagnetism, Superfluidity, edited by V. L. Ginzburg (MIR, Moscow), p. 242.

Ginzburg, V. L., and A. A. Sobyanin, 1988, Usp. Fiz. Nauk 154, 545 [Sov. Phys. Usp. 31, 289 (1988)].

Ginzburg, V. L., A. A. Sobyanin, and G. F. Zharkov, 1981, Phys. Lett. 87A, 107.

Ginzburg, V. L., and G. F. Zharkov, 1974, Pis'ma Zh. Eksp. Teor. Fiz. 20, 658 [JETP Lett. 20, 302 (1974)].

Ginzburg, V. L., and G. F. Zharkov, 1978, Usp. Fiz. Nauk 125, 19 [Sov. Phys. Usp. 21, 381 (1978)].

Ginzburg, V. L., G. F. Zharkov, and A. A. Sobyanin, 1974, Pis'ma Zh. Eksp. Teor. Fiz. 20, 223 [JETP Lett. 20, 97 (1974)].

Golder, L. S., N. Mulders, and G. Ahlers, 1992, J. Low Temp. Phys. 93, 131.

Gor'kov, L. P., 1959a, Zh. Eksp. Teor. Fiz. 36, 1918 [Sov. Phys. JETP 9, 1364 (1959)].

Gor'kov, L. P., 1959b, Zh. Eksp. Teor. Fiz. 37, 1407 [Sov. Phys. JETP 10, 998 (1960)].

Gorter, C. J., and H. Casimir, 1934, Phys. Z. 35, 963.

Landau, L. D., 1941, Zh. Eksp. Teor. Fiz. 11, 592 [J. Phys. (USSR) 5, 71 (1941)].

Lifshitz, E. M., and L. P. Pitaevskii, 1978, Statisticheskaya Fiz- ika (Statistical Physics), Pt. 2 (Nauka, Moscow) [translation: (Pergamon, Oxford, 1980)].

Little, W. A., 1964, Phys. Rev. 134, A1416.

London, F., and H. London, 1935a, Physica (Amsterdam) 2, 341.

London, F., and H. London, 1935b, Proc. R. Soc. London, Ser. A 149, 71.

London, F., and H. London, 1944, Proc. R. Soc. London, Ser. A 149, 71.

Maksimov, E. G., 2000, Usp. Fiz. Nauk 170, 1033 [Phys. Usp. 43, 965 (2000)].

Mamaladze, Y. G., 1967, Zh. Eksp. Teor. Fiz. 52, 729 [Sov. Phys. JETP 25, 479 (1967)].

Mamaladze, Y. G., 1968, Phys. Lett. 27A, 322.

Meissner, W. Z., 1927, Ges. Kältenindustr. 34, 197.

Ogg, R. A., Jr., 1946a, Phys. Rev. 69, 243.

Ogg, R. A., Jr., 1946b, Phys. Rev. 70, 93.

Pitaevskii, L., and S. Stringari, 2003, Bose-Einstein Condensation, International Series of Monographs on Physics No. 116 (Clarendon, Oxford).

Pitaevskii, L. P., 1958, Zh. Eksp. Teor. Fiz. 35, 408 [Sov. Phys. JETP 8, 282 (1959)].

Schafroth, M. R., 1954, Phys. Rev. 96, 1149.

Schafroth, M. R., 1955, Phys. Rev. 100, 463.

Selzer, P. M., and W. M. Fairbank, 1974, Phys. Lett. 48A, 279.

Shaplygin, I. S., B. G. Kakhan, and V. B. Lazarev, 1979, Zh.

Neorg. Khim. 24, 1476.

Shoenberg, D., 1965, Superconductivity, 3rd ed. (Cambridge University, Cambridge) [2nd ed. translated into Russian: (IL, Moscow, 1955)].

Tinkham, M., 1996, Introduction to Superconductivity (McGraw-Hill, New York).

Van Harlingen, D. J., 1982, Physica B \& C 109-110, 1710.

Waldram, J. R., 1996, Superconductivity of Metals and Cuprates (Institute of Physics, Bristol).

Wu, M. K., J. R . Ashburn, C. J. Torng, P. H. Hor, R. L. Meng, L. Gao, Z. J. Huang, Y. Q. Wang, and C. W. Chu, 1987, Phys. Rev. Lett. 58, 908.

Zharkov, G. F., 1958, Zh. Eksp. Teor. Fiz. 34, 412 [Sov. Phys. JETP 7, 278 (1958].

Zharkov, G. F., 1959, Zh. Eksp. Teor. Fiz. 37, 1784 [Sov. Phys. JETP 10, 1257 (1959)].

Zurek, W. H., 1996, Nature (London) 382, 296. 